\title{
A MIMO-OFDM Testbed for Wireless Local Area Networks
}

\author{
Albert Guillén i Fàbregas, ${ }^{1}$ Maxime Guillaud, ${ }^{2}$ Dirk T. M. Slock, ${ }^{2}$ Giuseppe Caire, ${ }^{3}$ Karine Gosse, ${ }^{4}$ \\ Stéphanie Rouquette, ${ }^{4}$ Alexandre Ribeiro Dias, ${ }^{4}$ Philippe Bernardin, ${ }^{4}$ Xavier Miet, ${ }^{4}$ Jean-Marc Conrat, ${ }^{5}$ \\ Yann Toutain, ${ }^{6}$ Alain Peden, ${ }^{7}$ and Zaiqing Li $^{7}$ \\ ${ }^{1}$ Institute for Telecommunications Research, University of South Australia, SPRI Building, Mawson Lakes Boulevard, \\ Mawson Lakes, SA 5095, Australia \\ ${ }^{2}$ Eurécom Institute, 2229 Route des Crêtes, 06904 Sophia Antipolis, France \\ ${ }^{3}$ Electrical Engineering Department, University of Southern California, 3740 McClintock Avenue, Los Angeles, CA 90089, USA \\ ${ }^{4}$ Motorola Labs, Centre de Recherche de Motorola, Espace Technologique Saint-Aubin, 91193 Gif-sur-Yvette, France \\ ${ }^{5}$ France Telecom R\&D, 3 avenue des Usines, 90007 Belfort, France \\ ${ }^{6}$ Antennessa, avenue la Perouse, 29280 Plouzane, France \\ ${ }^{7}$ École Nationale Supérieure des Télécommunications de Bretagne, Technopôle Brest-Iroise, 29238 Brest, France
}

Received 15 December 2004; Revised 11 May 2005; Accepted 21 June 2005

\begin{abstract}
We describe the design steps and final implementation of a MIMO OFDM prototype platform developed to enhance the performance of wireless LAN standards such as HiperLAN/2 and 802.11, using multiple transmit and multiple receive antennas. We first describe the channel measurement campaign used to characterize the indoor operational propagation environment, and analyze the influence of the channel on code design through a ray-tracing channel simulator. We also comment on some antenna and RF issues which are of importance for the final realization of the testbed. Multiple coding, decoding, and channel estimation strategies are discussed and their respective performance-complexity trade-offs are evaluated over the realistic channel obtained from the propagation studies. Finally, we present the design methodology, including cross-validation of the Matlab, C++, and VHDL components, and the final demonstrator architecture. We highlight the increased measured performance of the MIMO testbed over the single-antenna system.
\end{abstract}

Copyright (c) 2006 Albert Guillén i Fàbregas et al. This is an open access article distributed under the Creative Commons Attribution License, which permits unrestricted use, distribution, and reproduction in any medium, provided the original work is properly cited.

\section{INTRODUCTION}

Future wireless communication networks will need to support extremely high data rates in order to meet the rapidly growing demand for broadband applications such as highquality audio and video. Existing wireless communication technologies cannot efficiently support broadband data rates, due to their sensitivity to fading. Multiple antennas have recently emerged as a key technology in wireless communication systems for increasing both data rates and system performance. Ever since the landmark results by Telatar [1] and Foschini and Gans [2], there has been a growing interest in developing practical transmission schemes that exploit the extra spatial dimension to achieve reliable communication over multiple-input multiple-output (MIMO) channels. In particular, the application of such techniques to current wireless communication systems is of great interest. Highthroughput communication in indoor wireless local area networks (WLAN) is then a promising environment for the deployment of multiantenna equipments.

The main scope of this paper is to present the design steps leading to the demonstration of a software-radio testbed with multiple transmit $(\mathrm{Tx})$ and receive $(\mathrm{Rx})$ antennas, aiming at enhancing the performance of current WLAN standards. The expected benefits of multiple antennas are subject to the propagation conditions in wireless indoor local area transmissions. Therefore, the propagation environment must be carefully studied and analyzed before appropriate multipleantenna transmission techniques are devised and evaluated. In this regard, the scope of our work is larger than the typical prototyping efforts in that the project resulted in the production of an entirely custom demonstrator by the various industrial partners, including the algorithmic studies, the hardware handling the baseband processing, the RF components, up to the antennas, and the working real-time implementation of the coding and decoding algorithms. In particular, 
the channel characterization effort and the corresponding algorithmic adaptations proposed in this work, are typically absent from smaller-scale prototyping projects. This characterization was done by measuring the channel parameters in the frequency band of interest and developing channel models as well as corresponding channel simulation capabilities in order to produce typical channel impulse responses for algorithms evaluation. Then, using this preliminary information on propagation characteristics, various multiantenna processing techniques have been studied and compared according to performance versus complexity criteria. Similarly, the design and use of custom RF components and antennas are often replaced by channel simulators. Another major point in the validation of the testbed performance concerns the careful comparisons and cross-validations between hardware testbed performance and software simulation results. In the sequel, we give some details on the aforementioned steps and illustrate the design of the testbed as well as its performance improvement with respect to the single-antenna case.

The paper is organized as follows. Section 2 addresses the current status of WLAN specifications and the positioning of the paper in that respect. Section 2.1 presents the modeling of the propagation environment, and Section 2.2 shows the analysis of the propagation medium and its impact MIMO system design. Section 3 highlights some considerations about antennas and RF, in the view of future integration and production. Section 4 describes the several candidate algorithms for implementation on the testbed. In particular, multiple-antenna processing techniques in both open-loop and closed-loop modes and channel estimation algorithms are described. Section 5 provides details of the demonstration testbed as well as the performance improvement that was obtained thanks to the exploitation of multiple spatial streams. Finally, Section 6 presents the conclusions of this work.

\section{MIMO WLAN ENVIRONMENT}

After the success of WLAN products based on IEEE802.11b, the higher-rate WLAN solutions based on the IEEE802.11a/g standards have started to spread on the market. Such WLAN systems are based on orthogonal frequency-division multiplexing (OFDM), whereby the transmitted signal is constructed as the inverse Fourier transform of the actual constellation symbols to transmit. OFDM turns a frequencyselective channel into parallel flat-fading subchannels, each corresponding to a frequency subband of the total signal, with the benefit that simple equalization methods can be applied. Higher-order modulations, from BPSK up to (optional) 64-QAM enable to reach bit rates up to $54 \mathrm{Mbps}$, in a $20 \mathrm{MHz}$ band. These WLANs operate in the $2.4 \mathrm{GHz}$ industrial, scientific, and medical (ISM) band for the .11g OFDM solution and in the $5 \mathrm{GHz}$ unlicensed band for the .11a air interface. In this paper, we focus on the $5 \mathrm{GHz}$ band that represents the majority of the available spectrum.

Similar standardization efforts on WLAN systems have taken place in Europe and Japan simultaneously, giving rise to the ETSI HiperLAN/2 and ARIB MMAC specifications, that rely on exactly the same transmission principles as IEEE802.11a for the physical layer. However, because of the absence of existing products following the European specifications today, this paper refers to the IEEE802.11a context. In this work, some initial choices for the testbed were made based on HiperLAN/2-specific characteristics, such as the preambles used for channel estimation and synchronization. However, this does not affect the generality of the approach and the final performance results.

IEEE $802.11 \mathrm{a} / \mathrm{g}$ systems were initially developed taking into account a single-antenna constraint. Once the interest for MIMO systems gained in importance, multiple-antenna products appeared, which did not make full use of the multiple-antennas framework as envisioned by Telatar or Foschini. Essentially, they had to remain single-stream systems, that is, transmitting only one symbol per channel use, in order to remain compliant with the standard. In this case, the transmission does not benefit directly from the increased channel capacity promised by multiple antennas, but merely exploits the diversity in order to increase the link quality. Methods such as selection diversity or beamforming at the transmitter, and various types of combining or interference cancellation at the receiver were used.

Finally, with the clear recognition of the advantage of using multiple transmit and receiving antennas in terms of performance (link quality, range, bit rate), a new task group was created in 2003 within IEEE802.11, as .11n, with the charter of designing new WLAN specifications achieving up to $100 \mathrm{Mbps}$ on top of the media access control (MAC) layer in $20 \mathrm{MHz}$ band at $2.4 \mathrm{GHz}$ but mainly at $5 \mathrm{GHz}$. There, the key new technology introduced to enhance .11a performance is the MIMO signal processing capability. The other system parameters discussed today are very similar to the IEEE802.11a ones (64-point IFFT OFDM modulator for $20 \mathrm{MHz}$ band, baseline convolutional encoder). As a consequence, the work presented in this paper is perfectly relevant for the ongoing progress of standardization in the area of next-generation MIMO WLANs.

\subsection{Propagation environment modeling and simulation}

In order to evaluate the performance of radio access techniques, realistic channel modeling is required. To this end, a measurement campaign was performed in both outdoor (campus) and indoor operational environments at $5 \mathrm{GHz}$, in order to build a wideband 3D model of the channel (only the indoor measurements are presented here, further information about this campaign can be found in [3]). These measurements were further analyzed, in order to build a software channel simulator that can accurately replicate the environment of an indoor MIMO WLAN.

The MIMO channel was measured using two antenna arrays. A virtual array is formed by moving the Tx antenna (Figure 1(a)) on a grid composed of two horizontal and orthogonal 17-sensor linear antenna arrays (Figure 2(a)). The Tx antenna is omnidirectional and was set at $1.2 \mathrm{~m}$ above the floor corresponding to a mobile terminal (MT) location. The 


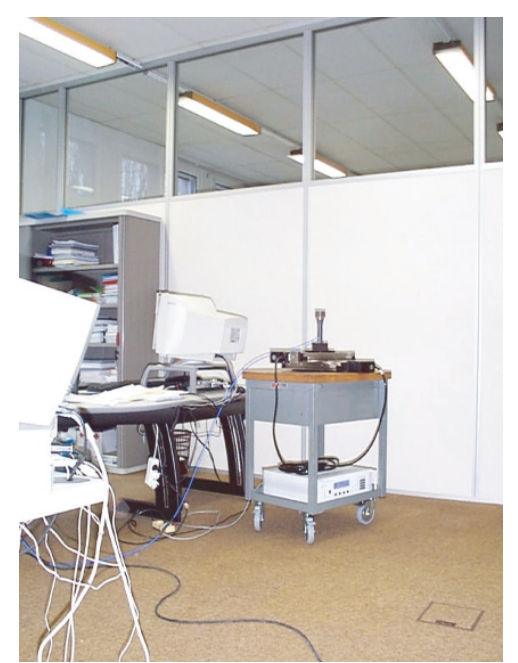

(a)

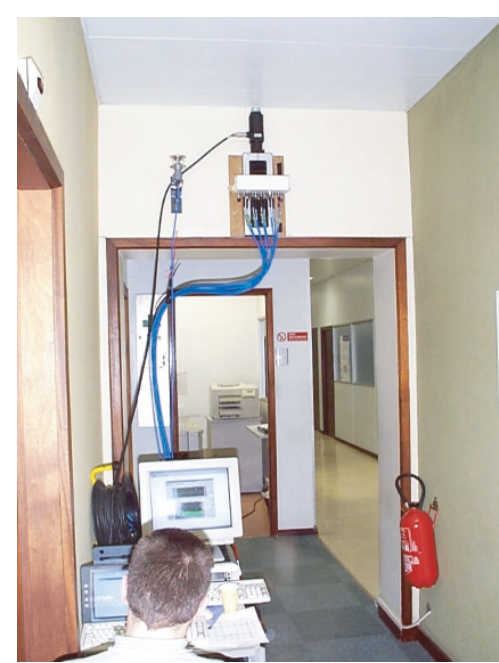

(b)

FIGURE 1: On-location channel sounding equipment: (a) mobile terminal and (b) access point.

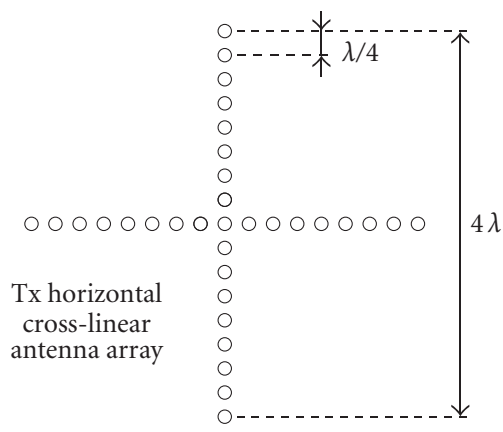

(a)

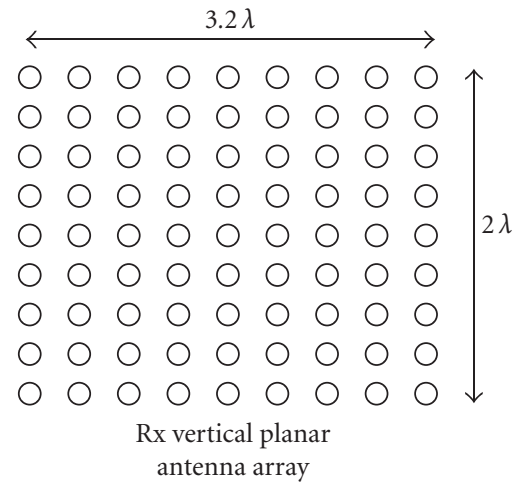

(b)

Figure 2: (a) Tx and (b) Rx antenna arrays configuration.

Rx antenna array (pictured in Figure $1(b)$ ) is an $9 \times 9$ vertical planar antenna array (Figure 2(b)). The horizontal and vertical apertures of the Rx antenna are, respectively, equal to $120^{\circ}$ and $90^{\circ}$. An additional fixed omnidirectional antenna is used to check the stationarity of the propagation channel during a full MIMO measurement. The Rx antenna was set at $2.6 \mathrm{~m}$ above the floor corresponding to an access point position. For each MT position, a full MIMO measurement consists of $34 \times 81$ complex channel impulse responses (CIR). CIRs were measured at $5.2 \mathrm{GHz}$ with a temporal resolution equal to 4 nanoseconds. The transmitted and received polarizations were vertical. The wideband measurements were performed by the Americc channel sounder developed by France Télécom R\&D.

Two Rx locations were chosen as representative of WLAN access point (AP) position. The first one was located in a corridor surrounded with rooms separated by very thin walls or glass doors. The second Rx position was located in a corridor surrounded with rooms separated by much thicker walls and wood doors. Twenty four transmitter locations were chosen as representative of MT positions (office, large meeting room, corridor, etc.). The maximum $\mathrm{Rx}$-Tx distance was $45 \mathrm{~m}$.

The data analysis phase consists of a space-time data processing applied to the measurements corresponding to each Tx-Rx location. The power angular profiles at the AP and MT were computed for each excess delay, using a standard beamforming algorithm. An example of 3D space-time diagrams at the AP is given in Figure 3. We can observe that the power delay profile (PDP) has the classic exponentially decaying shape often typical of indoor radio channels. Synthetic wideband and spatial parameters were also computed. For instance, the mean delay spread, AP azimuth spread, and AP elevation spread on the whole measurement campaign are, respectively, 19.2 nanoseconds, $20.2^{\circ}, 14.4^{\circ}$. The radio channel angular and frequency-selectivity analysis shows that the ETSI BRAN [4] models disagree with the results from the operational environments characterized in this 

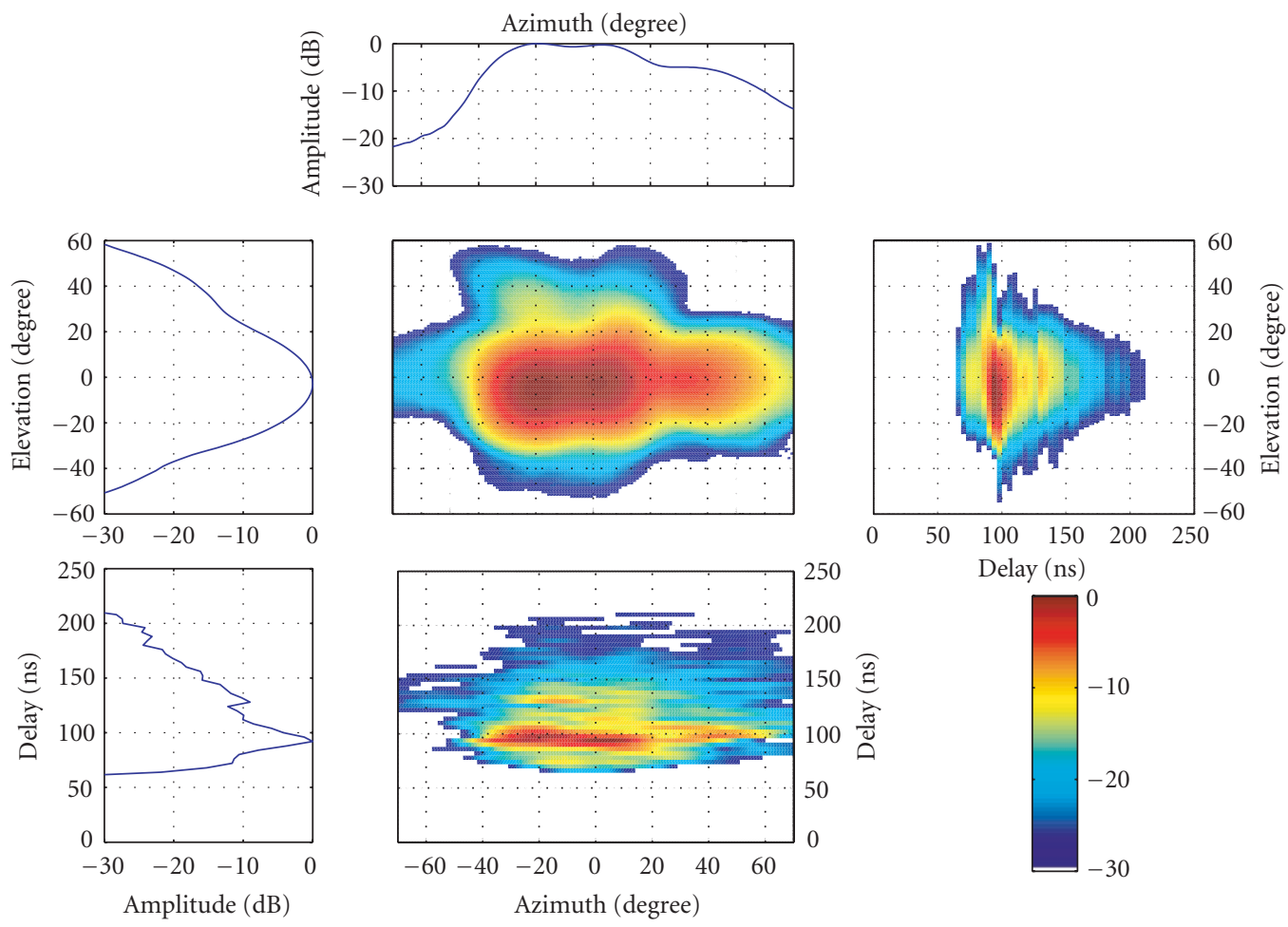

FIGURE 3: Space-time diagrams for a typical NLOS situation, $0^{\circ}$ corresponds to the antenna array boresight, delay spread, AP azimuth spread, AP elevation spread.

experimentation. In office environment, delay spread (DS) values are significantly smaller than the values recommended by Model A. The values of DS and AP azimuth spread (AS) strongly depend on the radio link configuration, which can be either line-of-sight (LOS) or non-line-of-sight (NLOS). No significant correlation was found between these two synthetic parameters and other parameters like $\mathrm{Tx}-\mathrm{Rx}$ distance or MT position (corridor, office, laboratory, etc.). Two wideband geometry-based propagation channel models are proposed for MIMO transmission simulations in indoor environment at 5.2 GHz: one for LOS situations and an another one for NLOS situations (Figure 1(b)). These models are wide sense stationary uncorrelated scattering (WSSUS) models and reproduce a selected MIMO measurement considered as typical. The developed models reproduce accurately the temporal dispersion and the angular dispersion seen at the AP. At the MT, the angular dispersion was only investigated by space-time diagrams visual inspection and a uniform 3D distribution was chosen to model the direction of arrival (DOA) at the MT. The MT DOA model could be refined by more accurate data analysis. Further information on geometrical models, and a selection of typical cases can be found in $[5,6]$.

The propagation channel models have been implemented using the Mascaraa ray-based radio channel software simulator [7], which simulates the polarized space-time behavior of the propagation channel. Therefore, the propagation channel is represented by a large set of rays with geometrical and propagation characteristics. Geometrical characteristics are delays and directions of arrival at the receiver and transmitter. The propagation characteristics are attenuations computed as functions of the transmitted and received polarization. The ray-based (so-called physical) approach has various advantages among which the most relevant are as follows:

(i) a set of rays includes all the information on the channel and every usually defined mathematical variable that can be obtained from the set of rays (channel impulse responses, Doppler, angular spectrum, correlation matrices, etc.),

(ii) most of the wideband channel models can be simulated with this simulation tool (tapped delay line models, tap directional models [8], scatterer or geometrical models, ray-tracing or ray-launching models [9]),

(iii) a set of rays models the propagation channel regardless of the antenna gains.

The channel simulator provides a large set of functionalities to facilitate the propagation models integration in simulation chains. The most important are the rays generation from a given channel model; the impulse response processing taking into account the $3 \mathrm{D}$ vectorial antenna pattern of each sensor; the convolution of the input signal with the simulated impulse response. The channel simulator is designed in a modular way and can be incorporated to link level simulation tools for radio-communication systems such as UMTS, HIPERLAN, WiFi, and so forth, with or without multiple antennas. 


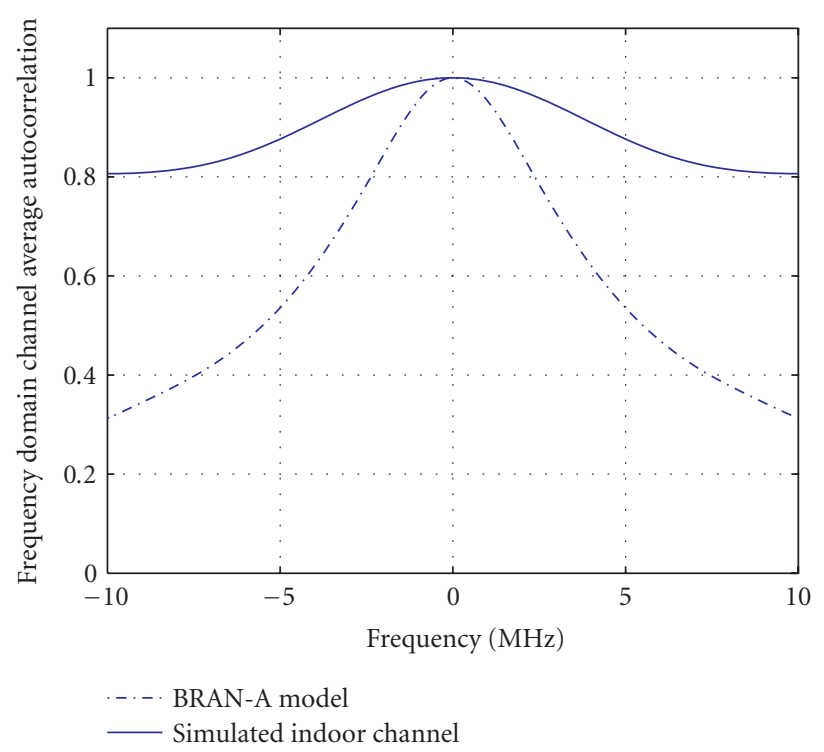

FIgURE 4: Correlation versus frequency spacing for the BRAN-A and the simulated WLAN channels.

\subsection{Impact of propagation characteristics on MIMO system design}

In order to extract design criteria for space-time and spacefrequency code design, simplifying assumptions on the channel statistics have to be made. In particular, a common assumption is that the channel can be described by a finite number of i.i.d., Gaussian random variables, this number being referred to as the channel diversity. Most codes are built to exploit the maximum diversity of the channel model. In order to gain a better understanding of the properties of a typical WLAN channel, an extensive measurement campaign was made, and a channel simulation tool was developed, as detailed in Section 2.1. We now present an analysis of the frequency and space diversity of the considered WLAN channel. The measurement-based channel simulator described above was used to generate channel realizations corresponding to an indoor, NLOS propagation model typical of an office environment, with $2 \mathrm{Tx}$ and $2 \mathrm{Rx}$ antennas. In an effort to remain close to the scenario of a mobile communicating with its AP, one side of the transmission link (the MT) has omnidirectional antennas, whereas the other side (the AP) has sectorial antennas with a $120^{\circ}$ aperture.

Statistical analysis of the simulated channel showed that, despite the rich multipath promised by the NLOS configuration, the indoor setting results in a small delay spreadof the order of two or three sample periods. Indeed, it was measured that the two strongest taps of the impulse response account on average for more than $90 \%$ of the power carried over the channel. The consequence is the limited frequency diversity available from such channel, that is, the strong correlation of the channel coefficients between subbands. This is depicted in Figure 4 as a function of their spacing in the frequency domain for a given transmit-receive antenna pair. It can be observed that correlation remains very high, even for frequencies situated far apart in the spectrum. The BRAN-A channel model has much larger frequency diversity, and as stated before, it does not accurately model a typical indoor scenario.

Another concern with MIMO systems is the antenna spacing necessary to ensure that different antennas actually yield uncorrelated channels. The AP-side (resp., mobile-side) correlation is defined as the off-diagonal coefficient of the normalized covariance matrix $\mathrm{E}\left[\mathbf{H}_{s}^{H} \mathbf{H}_{s}\right]$ (resp., $\mathrm{E}\left[\mathbf{H}_{s} \mathbf{H}_{s}^{H}\right]$ ), where $\mathbf{H}_{s}$ is the $2 \times 2$ MIMO channel associated with the strongest downlink path. At the MT side of the link, antenna separation of $\lambda / 2$ yields a correlation of 0.58 , whereas a separation of one wavelength $(\lambda=6 \mathrm{~cm}$ at $5 \mathrm{GHz})$ yields a correlation of 0.14 . At the AP, the correlation figures differ due to the different antenna diagrams. They are, respectively, $0.25,0.24$, and 0.25 for separations of $\lambda / 2, \lambda$, and $3 \lambda / 2$. The critical value is the MT antenna spacing, since the size constraints at the access point are presumably less stringent. A one-wavelength separation yields adequate channel correlation. According to [10], whereby the assumption of a normal distribution of scatterers of variance $\sigma_{\alpha}^{2}$ around the broadside axis yields a correlation $\rho\left(\Delta, \sigma_{\alpha}\right) \approx e^{(-1 / 2)\left(2 \pi \Delta \sigma_{\alpha}\right)^{2}}$, these values correspond to values of $\sigma_{\alpha}$ between $10^{\circ}$ and $20^{\circ}$. This result, associated with the low delay spread mentioned above, seems to denote a channel with few separable paths.

The ergodic capacity of a MIMO channel with $N_{T}$ transmit and $N_{T}$ receive antennas with complex Gaussian i.i.d. fading coefficients was shown by Telatar [1] to scale linearly with $\min \left(N_{T}, N_{R}\right)$ while the signal and noise powers remain constant. This is a very important property in favour of MIMO transmission, since not all system parameters have such an influence on the link capacity. Increasing the transmission power, for instance, only has a logarithmic influence on the capacity. We verified the Gaussianity of the channel coefficients by means of a $\chi^{2}$ analysis. Results revealed that they can be accurately modeled as Gaussian random variables. On the other hand, independence of the channels between different antenna pairs is by no means guaranteed in reality, since they essentially all depend on the same physical environment. This is a concern since correlation among channel coefficients can reduce the capacity. In extreme cases, such as the pinhole [11] (also called keyhole [12]) channel it was shown that the channel transfer matrix can be rank-deficient even though its coefficients are uncorrelated. In order to evaluate the impact of this phenomenon, we compared the capacity of the channel generated by the Mascaraa channel simulator, with that of a MIMO flat-fading channel with i.i.d. coefficients. This evaluation was done for a signal-to-noise ratio (SNR) of $30 \mathrm{~dB}$, for the cases $N_{R}=N_{T}=1,2$, and 4 antennas.

Note that both, the channel statistics and the nature of the channel are different for the i.i.d. Rayleigh and for the simulated channels. In fact, while one is i.i.d. in space, the other has correlation patterns at both, transmitter and receiver ends. Moreover, the i.i.d. channel is flat and the simulated channel has a nonnegligible delay spread. Figure 5(a) shows the evolution of the ergodic capacity and the $1 \%$ outage capacity versus the number of antennas. The ergodic 


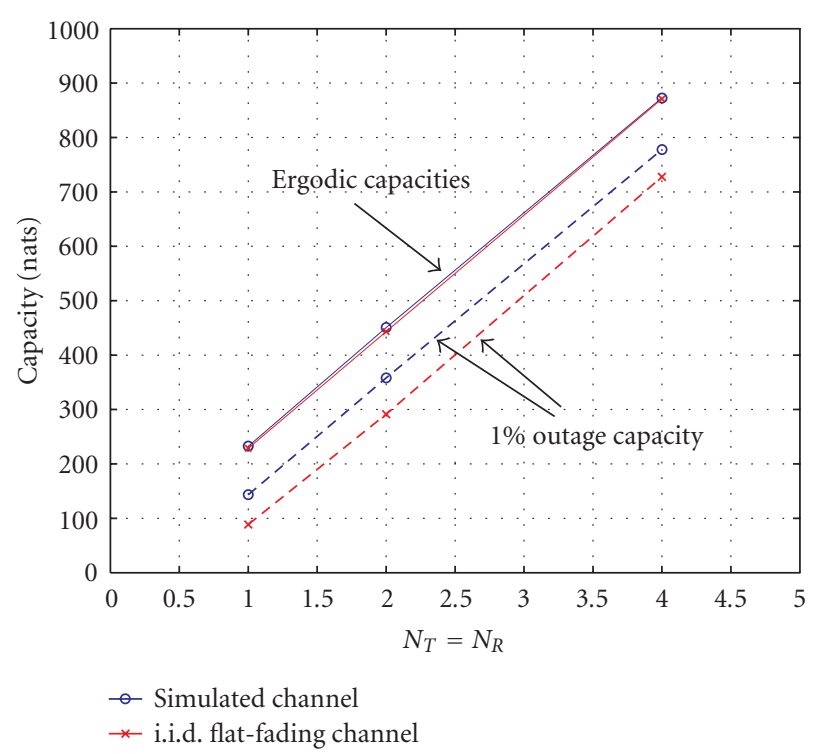

(a)

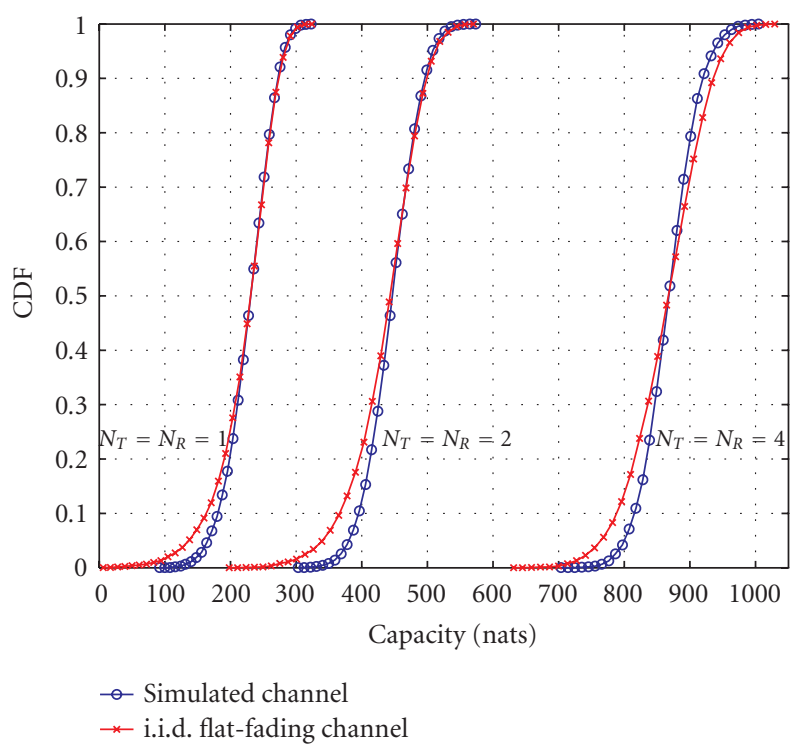

(b)

FIgURE 5: (a) Capacity evolution versus number of antennas and (b) capacity cumulative density functions.

capacity of the simulated channel follows closely the linear curve of the ideal (i.i.d. flat-fading) channel. Interestingly, the $1 \%$ outage capacity curves also show a linear increase with the number of antennas, with the simulated channel behaving better than the i.i.d. model. The capacity cumulative density function (CDFs) for these cases, depicted in Figure 5(b), provide more insight into this phenomenon: on average, in terms of capacity, the channel behaves close to a flat, Gaussian i.i.d. channel. A discrepancy between the curves is clearly visible in the lower probability range, that is, for the least likely cases, where the simulated channel is better than the flat i.i.d. channel. A likely explanation for this phenomenon is that the time-diversity of the channel, although reduced as we have seen before, mitigates the influence of the improbable draw of a very bad channel. A similar discrepancy is also visible at the high probability range, for the $N_{R}=N_{T}=4$ case. In this range, the simulated channel performs slightly worse than the ideal flat Gaussian i.i.d. model.

\section{ANTENNA AND RF CONSIDERATIONS}

The design of antenna arrays for MIMO systems can be optimized to maximally exploit the channel. This has been investigated in [13], where the considered optimization criterion was the ergodic capacity, in order to maximize bit rate. Such an optimization requires a doubly stochastic channel model: it should not only model the fast fading due to the superposition of many micropaths around a nominal path, but it should also describe the distribution of the nominal paths, since the multipath configuration in the actual deployment scenario is unpredictable. For these reasons, a stochastic channel model is required with a uniform distribution of angles of arrival and departure, possibly limited to a sector (oriented away from the wall) for the case of the AP. With such a uniform angular distribution, the pattern optimization for an array of (colocated) antennas leads to the following conclusions.

(i) The individual antenna responses should be orthogonal (uncorrelated).

(ii) The antennas jointly should form a uniform partitioning of the angular space (equal weight combining should lead to an omnidirectional response).

(iii) The antennas should introduce a sampling of the angles that is as dense and as uniform as possible. In this way, every antenna will contribute usefully, irrespective of the multipath scenario.

The orthogonality of antennas can be obtained by two mechanisms: either by amplitude variation or by phase variation. The first solution could be constructed on the basis of very thin sectoring into $N$ uniform sectors. Then, in order to construct an array with $M$ sensors, the diagram of one sensor consists of the union of the sectors obtained by taking one sector every $M$ (consecutive) sectors-this corresponds to a subsampling operation. In the case of phase variation, each sensor would be a spatial all-pass filter, characterized by its phase response. In practice though, it appears to be impossible to have phase variation without amplitude variation (similar to the minimum-phase property of linear systems). Therefore, the sector-based solution appears more realistic. A minimal solution would be to use one lobe or sector per sensor. However, such a solution is not very robust since it may be that very few paths pass in a given sector. To robustify this solution, a sensor with two lobes can be considered, centered around the same axis but oriented in the two opposite directions of this axis. If lobes with an opening of $60^{\circ}$ are considered, then the horizontal plane can be covered with three such sensors, as shown in Figure 6(a), with the angles 


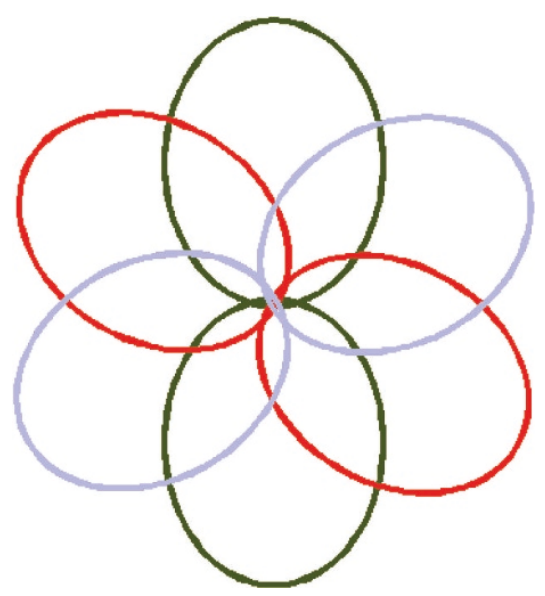

(a)

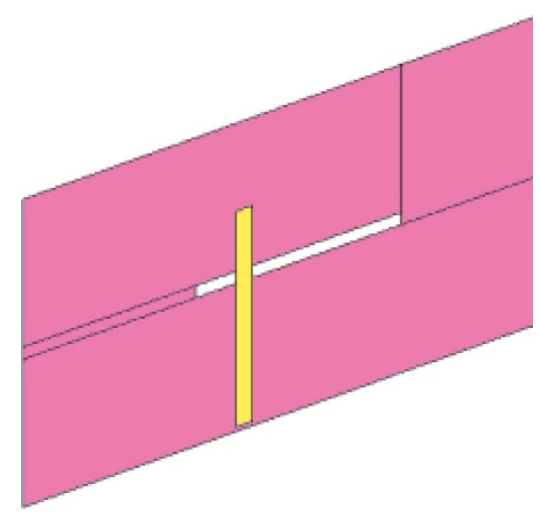

(b)

FIgURE 6: Pattern diversity with a double-lobe antenna array with 4 sensors. (a) 3 double-lobe responses and (b) slit antenna.

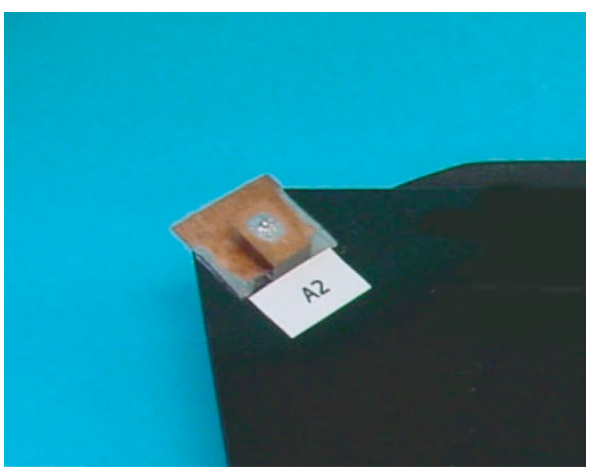

(a)

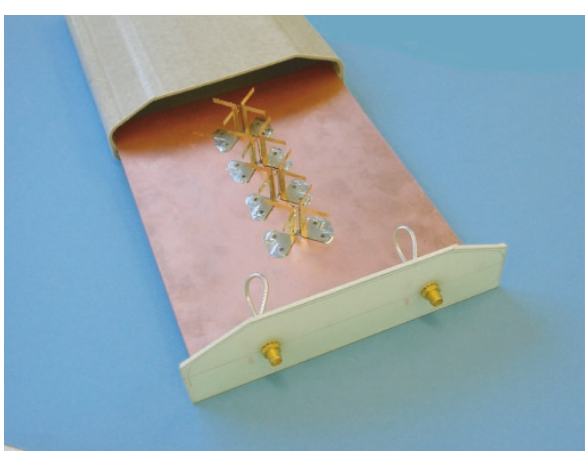

(b)

Figure 7: (a) Patch antennas for the MT and (b) antenna array for the AP.

between the three axes at $60^{\circ}$. A fourth such sensor can be added with a vertical axis. This would yield a system with 4 outputs, covering all directions with a certain robustness, due to the double sectors per sensor. An antenna whose diagram has the two opposite lobes mentioned above can be realized by a slit antenna, shown in Figure 6(b). The colocalization of four of such antennas poses a problem though.

The cost and performance issues associated with the mass-manufacturing of a multiple-antennas terminal were investigated. For example, the cost of integrating several antennas into a laptop computer includes the cost associated with the antennas themselves, and the modifications to the computer case, but also the need for additional radiofrequency (RF) circuitry if the antennas are to work simultaneously (in the case of antenna selection, this can be achieved by switching the antennas on a single RF front-end). The criteria for the antenna design include performance, integration, and cost. These parameters were evaluated for each proposed antenna design. Performance evaluation includes the antenna gain, power efficiency and standing wave ratio (SWR), the aperture of the radiation pattern, and, in the case of an antenna array, the coupling between antennas. Polarization diversity was also considered, although it was deemed too costly to exploit. All the antennas developed achieved a SWR lesser than 2 on the 5.15 to $5.35 \mathrm{GHz}$ band, and a radiation efficiency greater that $90 \%$.

An important effort has also been realized in order to propose some solutions that could easily be integrated in the mobile, and that could be produced in large scale in order to minimize the unit price. Among the considered antenna designs for the MT were monopole and dipole antennas, as well as a so-called patch antenna, made of folded stamped metal sheet. The latter, pictured in Figure 7(a), was selected for its sensitivity, higher than the monopole and dipole alternatives, and its low space selectivity. Despite these qualities, its manufacturing remains simple since it is made out of a single piece of metal sheet. Moreover, this technology enables the use of monolithic microwave integrated circuits (MMICs), which 
makes putting components on a printed circuit board (PCB) significantly easier and cheaper in terms of placing and soldering.

For the AP, in order to fit to the different scenarios of propagation (offices, open space), two types of antennas have been developed: single-element antennas and antenna arrays. Patch arrays were also considered, for their very high power efficiency, low manufacturing cost since the technology is similar to the PCB fabrication process. Their main limitation is their poor aperture (lower than $60^{\circ}$ ). Simple dipole and dipole arrays (pictured in Figure 7(b)), as well as dual-polarization dipole arrays were considered. Dipole arrays overcome the aperture limitation, and, in their dualpolarization version, can bring in polarization diversity with very good decoupling ( $>20 \mathrm{~dB}$ ) between polarizations. For the experimental phase of the project, the single-polarization dipole array technology was selected.

\section{ALGORITHMS}

This section describes some of the algorithms considered for implantation in the MIMO demonstrator. In particular, we consider space-frequency coding and decoding algorithms and channel estimation and we discuss their respective performance-complexity trade-offs. Due to the wide scope of this paper and for the sake of space limitation, we give only a qualitative description of the algorithms. The interested reader will find the appropriate references pointing to more detailed presentations.

\subsection{Coding and decoding}

Multiple antennas inherently increase the potential transmission rate and system diversity. We will then consider coding and decoding strategies that are able to benefit from the increased number of degrees of freedom in the system. This section briefly describes the coding and decoding algorithms studied for implementation in the MIMO demonstrator and compare them in terms of their performance versus complexity trade-off, with the aid of the specific ray-based channel simulator for WLAN described in Section 2.1. We first study open-loop techniques, for which the channel is assumed to be known at the receiver only. Then, we review several candidate strategies for the closed-loop case, where the channel is assumed to be known at both transmitter and receiver ends. All the algorithms described in this section, use the underlying convolutional code of rate $1 / 2$ and 64 states $(133,171)_{8}$ used in the HiperLAN/2 standard with packets of length 384 information bits.

\subsubsection{Open loop}

We here assume that the channel is perfectly known by the receiver only, and unknown to the transmitter. We consider concatenated space-frequency codes with symbol-based precoders, and direct space-frequency codes. In the first class, the bit stream is first encoded using the standard binary channel code, and mapped into constellation symbols, then parsed and possibly further linearly transformed to yield the actual signal to be transmitted. This class includes the spacetime block coding (STBC) methods developed by Alamouti [14], the ABBA method [15], spatial multiplexing associated to V-BLAST [16], and the linear precoding (LP) method developed in $[17,18]$. On the other hand, direct space-frequency codes divide the output of the binary code in subblocks, that are independently interleaved, modulated, and transmitted over a given (antenna, subcarrier) pair (see, e.g., [19-21] for more details). This approach is termed space-time bit-interleaved coded modulation (ST-BICM). For LP, iterative decoding is performed using minimum mean-squared error (MMSE) filtering and interference cancellation (IC). In the case of ST-BICM, simple interference cancellation using a matched filter (MF) is used.

\subsubsection{Closed loop}

Channel state information at the transmitter (CSIT) can provide substantial benefit in wireless communication systems compared to the no-CSIT case. CSIT can be obtained either through a feedback link, or by using the channel reciprocity property if both the uplink and downlink operate on the same frequency in time-division duplex (TDD) systems as HiperLAN/2.

We next consider coding schemes for multiple-antenna systems that exploit CSIT. In a nonergodic scenario with perfect CSIT, the optimal coding scheme (in the sense of minimizing the outage probability) performs the singular value decomposition (SVD) of the channel, reducing the MIMO channel to a set of noninterfering parallel channels, and computes the optimal power control rule. Such a coding scheme yields no-outage, that is, zero error probability at rates below the so called delayed-limited capacity [22]. However, due to the large representation size of the channel state and thus, the high induced complexity, we will focus on suboptimal schemes only. In particular, we consider the so-called transmit antenna array (TxAA) and selection diversity [23]. In essence, TxAA transmits over the largest eigenmode of the channel, while selection diversity transmits over the antenna whose channel has largest magnitude. As we will see, both perform very close.

\subsubsection{Numerical results}

In this section we show some selected numerical examples using the closed-loop and open-loop techniques described above using the ray-tracing simulator described in Section 2.1. In particular, we show the packet-error rate (PER) as a function of $E_{b} / N_{0}=\mathrm{SNR} / R$ per receive antenna, where $R$ is the spectral efficiency (in bits/s/Hz) and SNR is the total transmitted signal-to-noise ratio. Iterative methods employ the BCJR algorithm and five decoding iterations.

Figures 8(a) and 8(b) show the error probability for LP, V-BLAST, Alamouti, and ST-BICM with $N_{T}=N_{R}=2$ antennas and transmission rate $R=1,2 \mathrm{bit} / \mathrm{s} / \mathrm{Hz}$, respectively. 


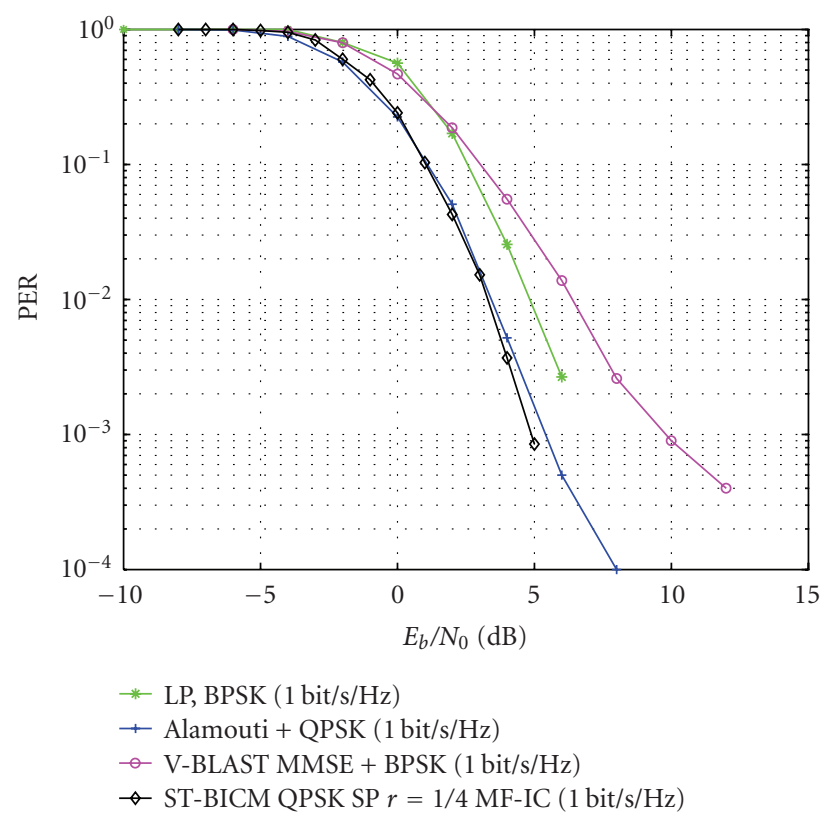

(a)

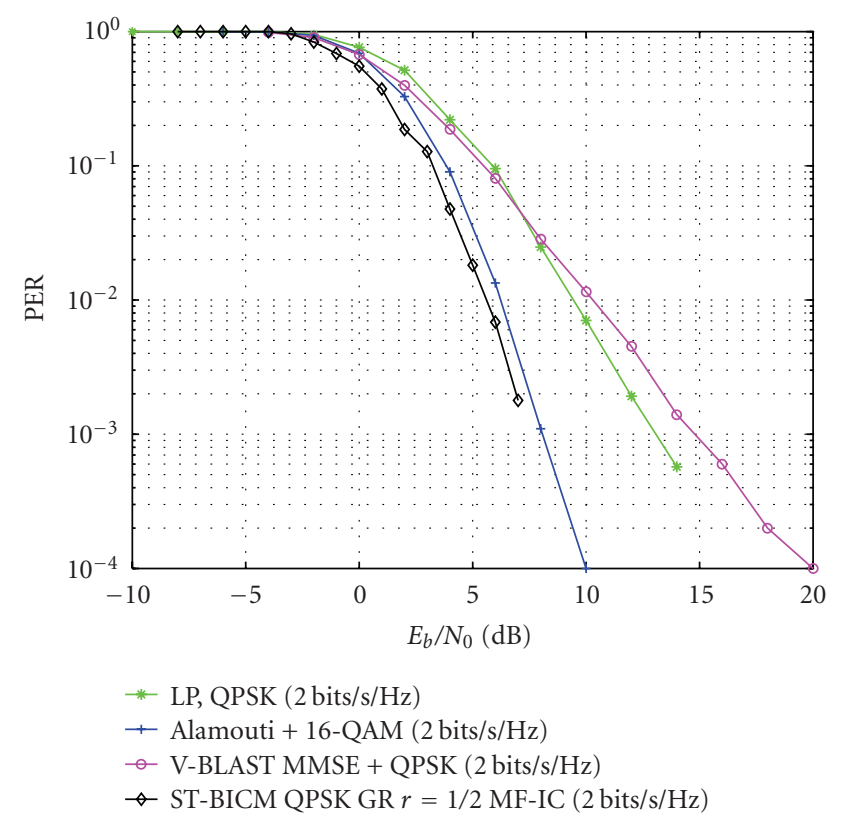

(b)

FIgure 8: Simulated PER for $N_{T}=N_{R}=2$, (a) $R=1 \mathrm{bit} / \mathrm{s} / \mathrm{Hz}$ and (b) $R=2 \mathrm{bits} / \mathrm{s} / \mathrm{Hz}$.

We first observe that ST-BICM performs slightly better than Alamouti, while the complexity of the latter scheme is significantly lower. In fact, the complexity of the Alamouti scheme is dominated by a single run of the Viterbi decoder. On the other hand, ST-BICM uses iterative interference with a matched filter, and thus, its complexity is dominated by the number of times (iterations) the BCJR algorithm is run. We also see that LP and V-BLAST do not always track the correct slope, exhibiting nonnegligible losses at PER of interest, for example, $10^{-2}$. For larger number of antennas, and higher data rates, ST-BICM provides larger performance advantage.

As stated before, selection diversity and TxAA are suboptimal, in the sense that they exploit CSIT to transmit only over a single mode of the channel, and thus, they implicitly induce a rate loss of a factor $N_{T}$. In fact, the weighting operation can be seen as a linear modification of the channel, creating a new virtual channel of lower capacity. Figure 9(a) shows the CDF of the mutual information for the above methods with $N_{T}=N_{R}=4$, perfect CSIT, and SNR $=3 \mathrm{~dB}$. For the sake of reference, we show the open-loop case (no CSIT) and two water-filling solutions with short-term power constraint: global water-filling over the entire dimension of $\mathbf{H}$ and a pertone water-filling. The global water-filling solution is the optimal allocation of power inside an OFDM symbol with instantaneous power constraint [1]. Note that, under shortterm power constraint, CSIT is only relevant at low SNR. Due to the possibly large dimension of the problem, global waterfilling can be complex. Interestingly, the lower-complexity per-tone water-filling, where the power is the same in each frequency subband, and spatial water-filling is used independently in each subband, is near-optimal.
Obviously, TxAA and selection diversity are shown to be largely inferior, due to their rate-one nature. Moreover, we observe a the degradation incurred by selection diversity, due to its suboptimality with respect to TxAA.

Figure 9(b) shows the error probability with perfect CSIT of selection diversity and TxAA with $N_{T}=2$ and $N_{R}=1,2,4$ antennas and transmission rate $R=1 \mathrm{bit} / \mathrm{s} / \mathrm{Hz}$. As expected by the above discussion on outage probability, TxAA outperforms selection diversity. However, as selection diversity is much less complex and more robust to channel estimation errors, it is a good candidate for practical implementation. Moreover, as a rate-one scheme, Figure 8(a), selection diversity provides a $1 \mathrm{~dB}$ gain with respect to Alamouti and STBICM.

\subsection{Synchronization and frequency offset estimation}

Synchronization of an MTMR OFDM system is achieved using training symbols which are simultaneously transmitted from all transmit antennas at the beginning of each frame. The HiperLAN/2 broadcast channel $(\mathrm{BCH})$ preamble is described in Figure 10. This preamble is divided into 3 fields: the A-field, for coarse AGC and frame detection, the B-field, for AGC setting, fine time synchronization, and frequency offset estimation, and the C-field, which is dedicated to channel estimation and frequency offset estimation refinement. HiperLAN/2 usually sets the channel estimation training sequences $\mathrm{C} 1$ and $\mathrm{C} 2$ to be equal. However, as will be shown next, using different sequences may impact the performance and complexity of channel estimators for MIMO channels. Again, any reference to the HiperLAN/2 standard in this 


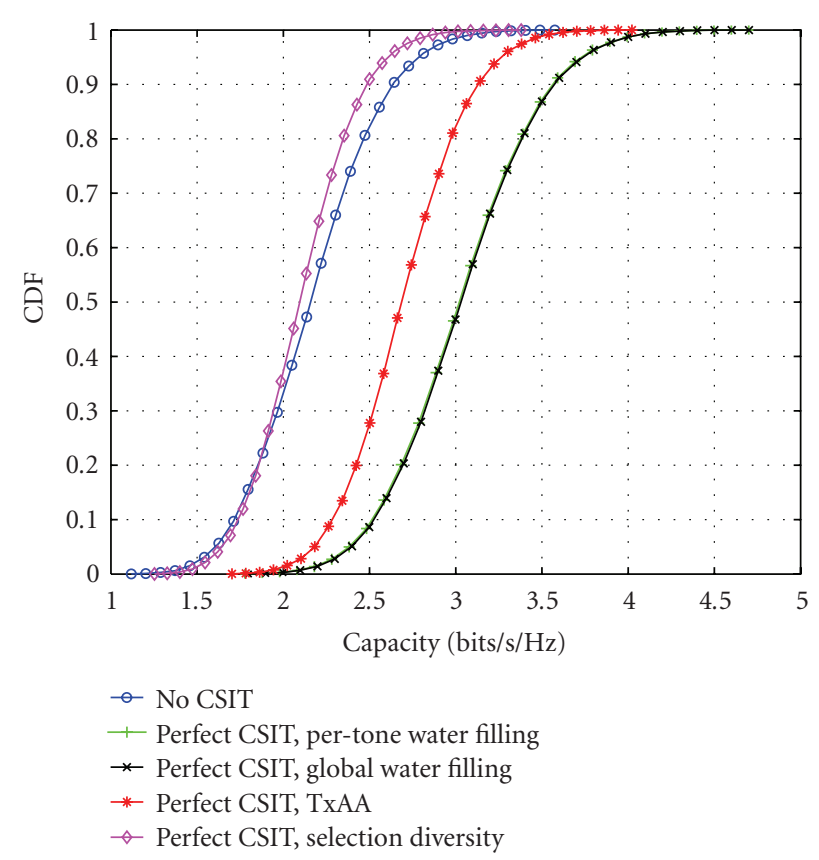

(a)

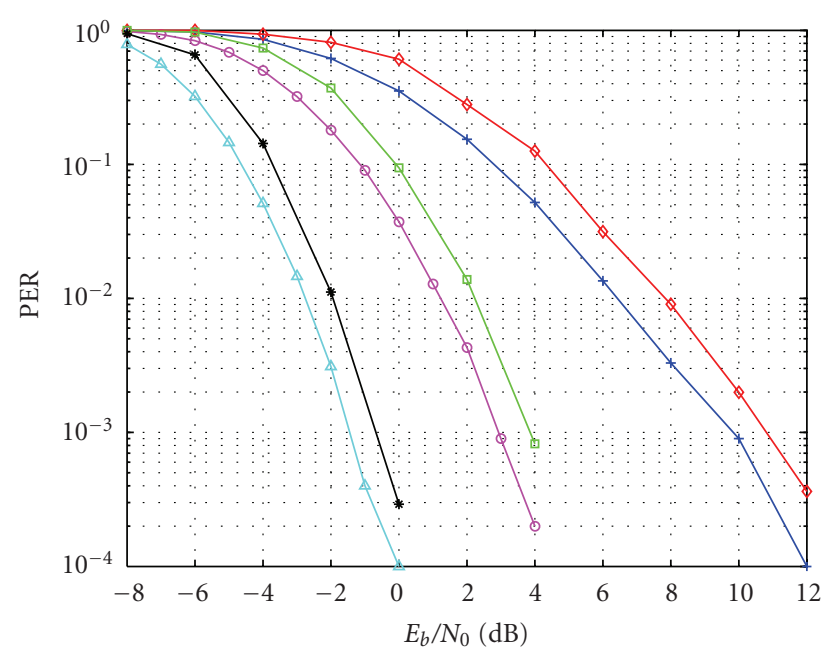

$$
\begin{aligned}
& \rightarrow \text { TxAA } 2 \times 1 \\
& - \text { TxAA } 2 \times 2 \\
& -\triangle \text { TxAA } 2 \times 4 \\
& \rightarrow \text { SD } 2 \times 1 \\
& - \text { SD } 2 \times 2 \\
& - \text { SD } 2 \times 4
\end{aligned}
$$

\begin{tabular}{|c|c|c|c|c|}
\hline A-field & B-field & C32 & C64 & C64 \\
\hline $\begin{array}{l}\text { AGC and coarse } \\
\text { time synchronization }\end{array}$ & \multicolumn{2}{|c|}{$\begin{array}{l}\text { AGC setting, frequency } \\
\text { offset estimation, and time } \\
\text { synchronization }\end{array}$} & \multicolumn{2}{|c|}{ Channel estimation } \\
\hline
\end{tabular}

(b)

FIgURE 9: (a) Closed loop: mutual information CDF for various closed-loop methods on a $4 \times 4$ channel at $-3 \mathrm{~dB}$ SNR and (b) PER of selection diversity and TxAA for $N_{T}=2$ and $R=1 \mathrm{bit} / \mathrm{s} / \mathrm{Hz}$.

Figure 10: BCH preamble.

section should be interpreted as a reference to a fairly arbitrary OFDM WLAN system for which the values of a number of parameters are chosen as in the HiperLAN/2 or equivalently IEEE802.11a standards.

A number of different training signal sequences have been proposed for $(1 \times 1)$ OFDM systems such as HiperLAN/2. However, those training symbols, also called preambles, for synchronization and channel estimation, cannot be applied without care, and potential modifications, to multiple transmit and receive antennas systems since autocorrelation properties of these sequences are altered by the contribution of all the channels in the received signal. Synchronization consists of a four-step process: when the MT is switched on, it has to

(1) coarsely detect the start of a new frame;

(2) achieve time synchronization;

(3) estimate and compensate frequency offset in the time domain;
(4) estimate the channel in the frequency domain and perform equalization.

Different parts of the OFDM preamble (Figure 10) are used at each step: the A-field is used for coarse AGC and frame detection, the B-field is used for AGC setting, fine time synchronization, and frequency offset estimation, and the Cfield for channel estimation and frequency offset estimation refinement. The synchronization scheme selected for implementation is based on parallel simultaneous transmission of the the same A-, B-, and C-fields by all antennas. Therefore, the autocorrelation properties of the preamble (Figure 11) remain unchanged at the receiver. We assume that the signals are received almost simultaneously (with respect to the sampling frequency), that is, the time instant for received signals is the same, which is a reasonable assumption given the small antenna separation. We also exploit the fact that a single oscillator is used for both transmit antennas and another oscillator for both receive antennas, that is, the frequency 

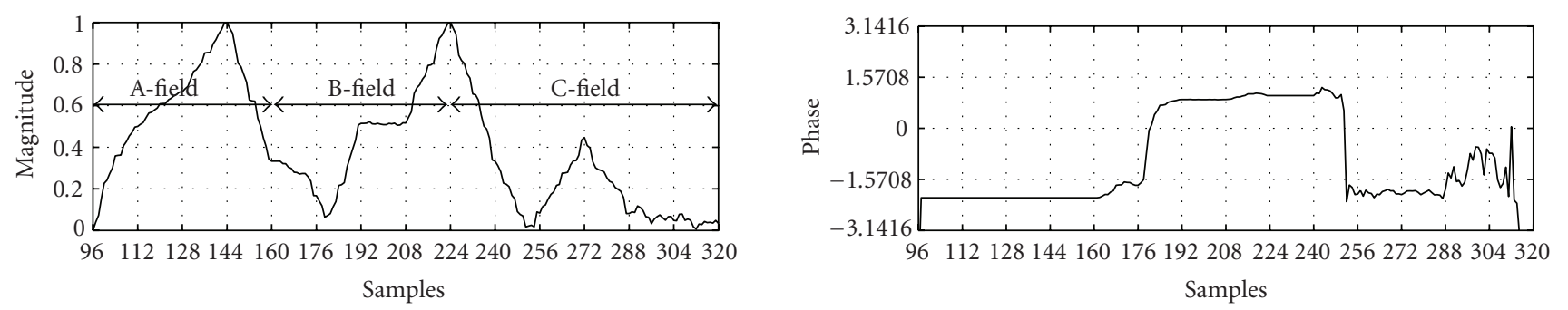

FIGURE 11: Autocorrelation of BCCH preamble with a $200 \mathrm{kHz}$ frequency offset.

offset is the same for all transmit-receive antenna pairs. In our demonstrator, the number of unknowns that need to be estimated is therefore exactly the same as in the singleantenna case, and we can thus apply the single-antenna synchronization algorithms.

Frequency synchronization consists in finding an estimate of the difference in the frequencies between the transmitter and receiver local oscillators. Frequency offset estimation in OFDM is critical since any frequency offset causes a loss of subchannel orthogonality, which results in intercarrier interference (ICI) and hence performance degradation. The two main frequency offsets to estimate are carrier and clock offsets. Due to oscillator inaccuracies, the received spectrum is usually slightly shifted to the right or left: this is the so-called carrier frequency offset or frequency offset. The second error to estimate is due to the drift of the local oscillator at the receiver: this is the clock frequency offset. The method considered for carrier frequency offset estimation exploits autocorrelation phase properties. Once the frequency offset has been estimated, it is then compensated in time domain on the C-field in order to perform channel estimation. The selected method is summarized below.

(i) Coarse frequency synchronization: the phase of B-field peak provides a rough estimate of the frequency offset within the interval $[-625 \mathrm{kHz} ; 625 \mathrm{kHz}$ [, since the Bfield sequences length is a quarter symbol (autocorrelation on 16 samples); there is no ambiguity since the maximum frequency offset defined in the standard is $200 \mathrm{kHz}$. However, the degradation of the SNR due to ICI can still be important.

(ii) Fine frequency synchronization: the C-field sequence length is one full symbol. If only the C-field was used, there would be an ambiguity in the frequency offset. But thanks to the B-field rough estimate, this ambiguity can be resolved, and the SNR degradation becomes acceptable.

\subsection{Channel estimation}

In this section, we discuss low-complexity channel estimation algorithms suitable for implantation in the MIMO testbed. Many pilot-aided channel estimation techniques exist for single-antenna OFDM-based systems. When directly exported to the MIMO case, such techniques fail, since the signals coming from the different transmit antennas interfere at the receiver end. Moreover, the number of parameters to be estimated can be remarkably larger in the MIMO case, inducing more complex estimators. More explicitly, in a single-antenna OFDM system, the number of parameters to be estimated is $N_{C}$, while in a multiple-antenna system it is $N_{C} N_{T} N_{R}$, though the amount of data also increases by a factor $N_{R}$. In this section, we describe how to extend SISO techniques to the MIMO case while keeping complexity low.

Sending the same pilot symbols simultaneously from all Tx antennas fails to properly sound the channel, since it only excites it along a single spatial direction. Therefore, repeating the same pilot will not bring significant improvement. On the other hand, joint design of the pilot symbols can be important. In particular, using orthogonal designs as pilots $[14,24,25]$, and using a training sequence of length $L \geq N_{T}$, followed by a least-squares estimate in the frequency domain, yields an accurate estimate within reasonable complexity. This is possibly the simplest applicable estimation algorithm. Bayesian linear MMSE estimation techniques can also be used. They rely on the cross-correlation matrix between the channel and the received signal and the autocorrelation matrix of the received signal, which in turn depend on the autocorrelation matrix of the channel elements. These matrices have to be estimated and hence the MMSE methods are more complex to implement.

In order to reduce the number of parameters to be estimated, one can resort to time-domain channel estimation techniques. In this case, only the coefficients of the channel impulse response (assumed to be much less than $N_{C}$ ) have to be estimated. A simple multiplication by the Fourier transform matrix will return the frequency-domain channel parameters. Unfortunately, however, such techniques yield a larger overall complexity (see [25] for details). Further details on these aspects and their exploitation can be found in [26].

Figure 12 illustrates the performance of several channel estimators in a MIMO channel with $N_{T}=N_{R}=2$ antennas using the Alamouti scheme, the standard 64 states convolutional code with QPSK modulation (the pilot symbols are orthogonal and use BPSK) in the BRAN-A channel model [4]. It can be observed that while the frequency-domain LS is $2.5 \mathrm{~dB}$ away from the perfect channel knowledge case, its time-domain version (more complex) performs almost as well as the MMSE. 


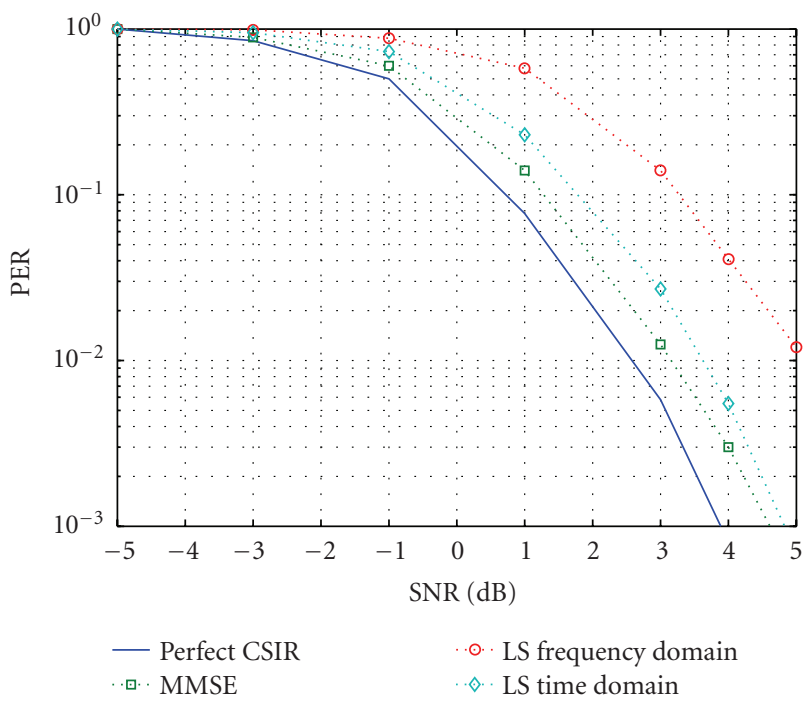

FIgURE 12: Performance of different channel estimators with the BRAN-A channel model.

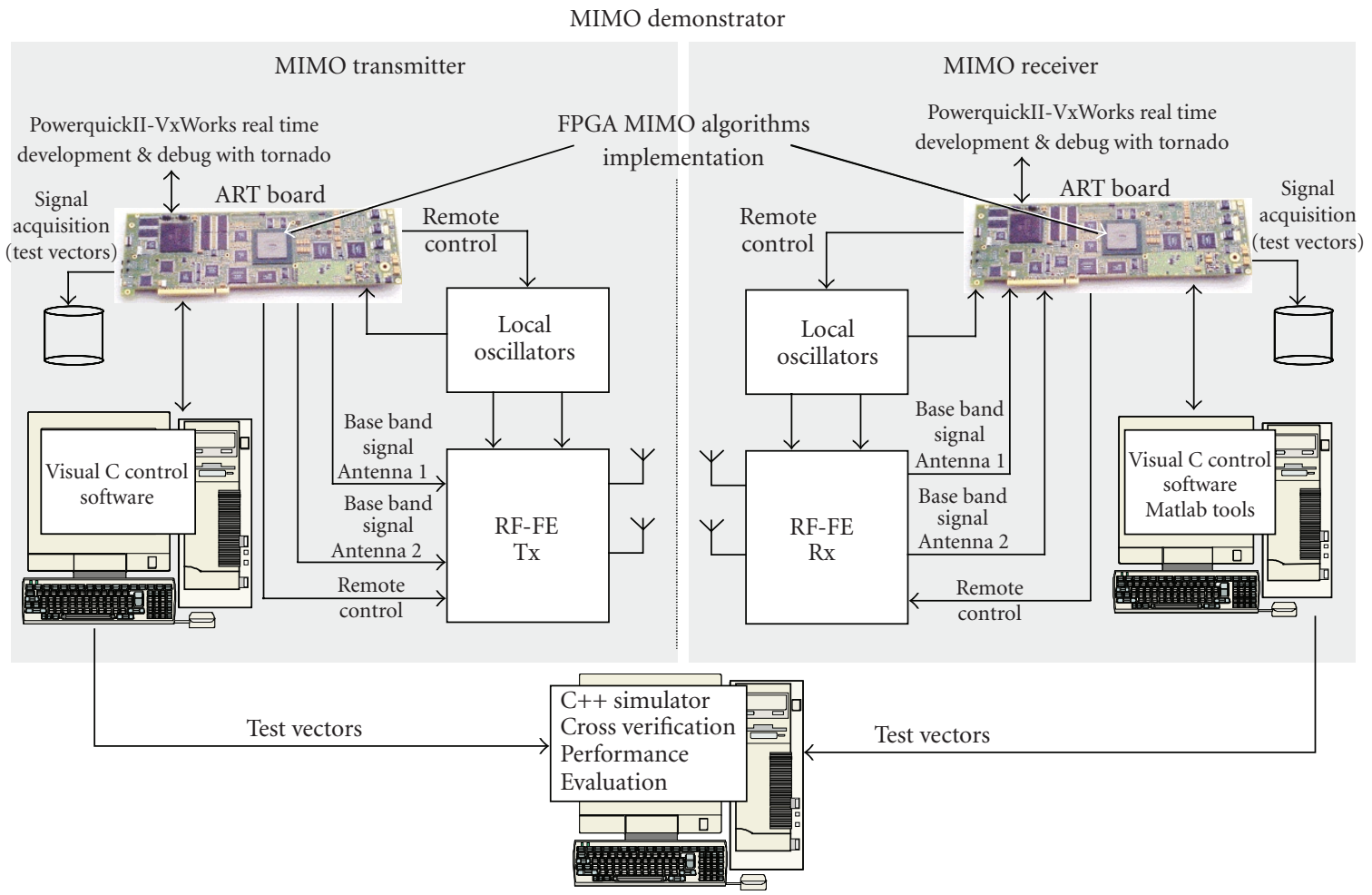

FIGURE 13: Multiple-antenna testbed and performance assessment.

\section{MIMO-OFDM DEMONSTRATION TESTBED}

In this section we describe in some detail the implementation aspects of the testbed. The main goal of the testbed development is the validation of the performance of MIMO algorithmic approaches in real-time hardware, in order to highlight the potential improvements brought by different modes implemented, in comparison with the single-antenna case. We also highlight the methodology used in the testbed development, using an FPGA for digital processing. The full setup is depicted in Figure 13. The steps followed include full $\mathrm{C}++$ floating-point and fixed-point simulations and crossvalidations with VHDL code simulations. Performance results have been checked at each step. 
After the algorithmic study, a limited set of MIMO algorithms were selected for further implementation, based on the following criteria: (1) best complexity versus performance trade-off, assessed by high-level simulations, (2) acceptable level of complexity, given the available computational resources, and (3) embedded reconfigurability with a single-antenna fallback mode. In the view of the above, the selected solution for demonstration embeds up to $N_{T}=2 \mathrm{Tx}$ and $N_{R}=2 \mathrm{Rx}$ antennas. The modulation is based on the Alamouti space-time block code for the $2 \mathrm{Tx}$ modes, and on per-subcarrier maximum ratio combining (MRC) for the 2 $\mathrm{Rx}$ modes, since it has very low complexity and it still offers excellent performance on the realistic channel, as shown in Section 4. The demonstrated system also embeds an OFDM modulator, with modulation schemes ranging from BPSK to 16-QAM, and forward error correction mechanisms based on the HiperLAN/2 (or equivalently, IEEE802.11a) specifications, as well as synchronization and frequency-domain LS channel estimation procedures. This results in a point-topoint setup, with embedded performance evaluation mechanisms (such as bit error rate computation), usable in different locations and scenarios.

\subsection{Hardware characteristics}

\section{Baseband platform}

The algorithms have been ported to the advanced research technologies (ART) platform. The hardware used in this demonstration platform consists of a radio module and a baseband processing card. The baseband processing card consists of a general-purpose microprocessor, for the non-time-critical MAC code, a large FPGA for the timecritical data link control (DLC) and physical (PHY) layer functions, and the analog components needed to interface to the RF subsystems. Explicitly, there are two digital-toanalog converters (DACs) and two analog-to-digital converters (ADCs) on each baseband card. The FPGA is an ALTERA EP20K1500EBC652-1X (1.5 million gates). It is interfaced with two 12-bit ADCs and two 12-bit DACs (resp., Analog Devices AD9432BST-105 and AD9752ARU). The FPGA transmitter and receiver designs include the automatic gain control (AGC), the synchronization, the OFDM engine, and the digital I/Q modulator/demodulator. The baseband processor is connected to the radio front-end via two AD/DA converters of 12 bits each, sampled at $80 \mathrm{MHz}$. The transmitter and receiver designs are based on proprietary blocks written in VHDL. We choose the development to be performed within three main steps: algorithm-level simulation (Matlab \& $\mathrm{C}++$ ), then VHDL simulation and FPGA implementation. The validation of the test bed relies on a crossverification: simulation and implementation environments are compared via common scenarios and bit-accurate test vectors. The baseband implementation of the receiver is split into two designs: one is dedicated to the testbed signal acquisition while the other one helps to validate the digital loop-back process of the cyclic prefix OFDM (CP-OFDM) modulator. In addition, the transmitter uses a frame scenario player relying on an instruction pipelined sequencer. The system can handle BSPK, QPSK, and 16-QAM constellations. The PCI baseband board is controlled by a $C$ application under MS-Windows. It allows to set any baseband parameters and frame scenario. On the receiver side, the signal acquisition and analysis is handled by an additional Matlab environment including the OFDM demodulator chain for both, the CP-OFDM and PRP-OFDM modes. All commands and transfers initiated by the host PC are relayed by the PowerPC available on the board; we choose to run this CPU with the real-time operating system (RTOS) VxWorks. The signal acquisition modules handled by the PowerPC allow to capture approximately six OFDM frames of 256 data symbols each.

\section{RF front-ends}

The architecture of the receiver RF front-end is based on classical heterodyne (double IF-intermediate frequency) conversion. It performs the frequency down-conversion only, since the demodulation is performed digitally in baseband (digital I/Q separation) on a signal sampled at $80 \mathrm{MHz}$, with a lower IF at $20 \mathrm{MHz}$. At the transmitter, the frequency upconversion is achieved with the same principle. The obvious advantage of this scheme is that only a single baseband processing card is needed for the 2 radios. A digital antialiasing filter is needed in the receiver to prevent the real and imaginary parts of the signal from being mixed. If an adjacent channel rejection filter is also needed, it must be constructed as a band pass filter in at a low IF frequency in analog. This design is difficult to realize, and thus "digital I and Q" imposes that the adjacent channel filter must be performed in the digital domain. Although analog I/Q would allow to use a lower sampling clock, and therefore reduce clock jitter and nonlinearities due to skew, it requires the compensation of the I/Q imbalances, both in amplitude and phase, introduced by the separated I/Q processing in the analog domain. Furthermore, using digital I/Q involves only one DAC and ADC per radio channel, versus one for each of the I and Q channels in the analog version. Two local oscillators are used for the frequency conversions (the same for up- and down-conversions) and are based on programmable PLL synthesizers which enable channel selection over the HiperLAN/2 band. The demonstrator uses the lower HiperLAN/2 band (5.180-5.320 GHz), in which the effective isotropic radiated power (EIRP) is limited to $23 \mathrm{dBm}$. The maximum receiving power to be supported according to the specifications is $-30 \mathrm{dBm}$ (class 2 receiver). The measured performances of the receiver fulfill the sensitivity and SNR specifications for the various data rates (from $6 \mathrm{Mbit} / \mathrm{s}$ to $54 \mathrm{Mbit} / \mathrm{s})$. The demonstrator, pictured in Figure 14(a), is built upon purchased components and circuits that were mounted on dielectric substrates. Some circuits were specifically designed for the RF architecture such as local oscillators, image rejection filter, and automatic gain control (AGC) amplifiers at the first and second IF. Compliant with the IEEE802.11a/HiperLAN/2 definition of the PHY layer, the 


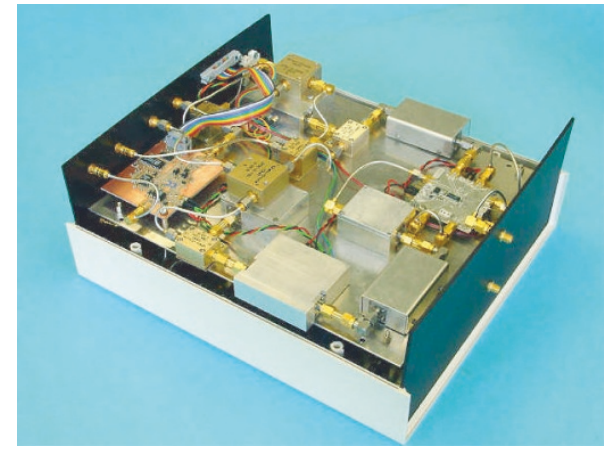

(a)

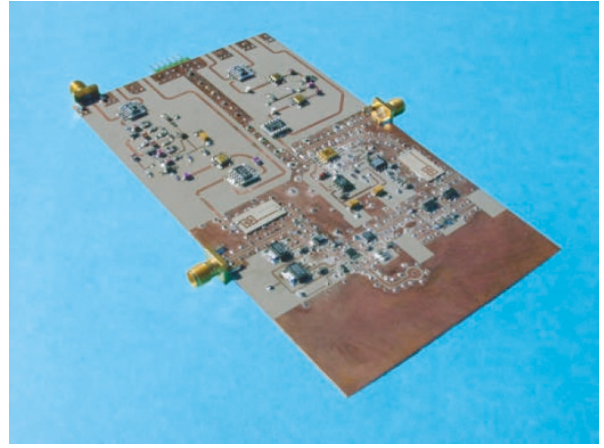

(b)

FIgURE 14: RF front-ends. (a) RF-FE stage for $2 \mathrm{Tx} / \mathrm{Rx}$ modules. (b) Integrated RF front-end PCB.

demonstrator architecture of the $5 \mathrm{GHz}$ part uses an intermediate fixed frequency at $930 \mathrm{MHz}$ and a voltage-controlled oscillator (VCO) at $4.2 \mathrm{GHz}$ to address several $20 \mathrm{MHz}$ channels between $5.15 \mathrm{GHz}$ and $5.35 \mathrm{GHz}$. On the receiver side, the chain embeds an AGC with a dynamic range of $80 \mathrm{~dB}$ that pushes the overall sensitivity below $-85 \mathrm{dBm}$. On the transmitter side, the output power is controlled by an attenuator with $15 \mathrm{~dB}$ of dynamic range followed by two power amplifiers (PA) in order to maintain the output power between $-13 \mathrm{dBm}$ and $+10 \mathrm{dBm}$.

In order to reduce cost and more specifically size and volume, the integration of the RF front-end close to the antenna is essential. An RF front-end was designed specifically for this project. Almost all of the RF and IF functions of a single front-end were implemented on one side of a $10 \mathrm{~cm} \times 15 \mathrm{~cm}$ double-side PCB, with the DC power supply occupying the other side, together with the synthesizers and the second IF AGC amplifier. In order to achieve this, new designs of some circuits were performed: the image rejection filter was designed on a separate high permittivity dielectric substrate and then mounted on the PCB, the transmitter power amplifier, the receiver low-noise amplifier, and the switch were mounted using separate packaging integrated circuits. The sheet metal antenna is reported on the opposite side of the RF circuits. The integrated front-end circuit board is depicted in Figure 14(b).

\section{Antenna characteristics}

The antennas selected for the demonstration platform address different sets of requirements, and thus have different characteristics at the mobile terminal and the access point (see Figure 7). At the MT, we selected low-cost sheet metal patch antennas to be integrated into the lid of a laptop, on the corners, so as to get maximum space diversity (see Figure $7(\mathrm{a})$ ). They are also oriented so as to get polarization diversity. The radiation pattern is omnidirectional, and such antennas present a good radiation efficiency, but a poor isolation in cross-polarization configuration. It was verified that in all cases, the coupling between elements is below
$-30 \mathrm{~dB}$. At the AP, we selected again a low-cost sheet metal antenna, as baseline single element, having a radiation pattern with $110^{\circ} 3 \mathrm{~dB}$ aperture in the vertical plane, and $90^{\circ}$ in the horizontal plane, and the two antennas used also present polarization diversity with $15 \mathrm{~dB}$ decoupling. The antenna gain here is $6 \mathrm{dBi}$. In addition, we considered a second possibility, which consists in using the baseline elements described above within an antenna array, having this time a radiation pattern of $\left(110^{\circ}, 30^{\circ}\right)$ and a $9 \mathrm{dBi}$ gain. Again, it is possible to use polarization diversity in addition to space diversity, and the antennas are mounted on a board, so that they can be tilted (see Figure 7(b)).

\subsection{Development methodology}

The VHDL design is validated through the following steps:

(1) test VHDL-blocks using software simulation (e.g., (i)FFT, Viterbi decoder, synchronization, etc.); all output vectors are compared to bit-accurate test vectors in $\mathrm{C}++$ and/or Matlab;

(2) synthesize the blocks and check their behavior within the FPGA on the platform. During this process, the FPGA outputs must correspond exactly to the results of the bit-accurate simulations. This approach is valid for the Tx as well as for the Rx: concerning the Tx, the signals at the output of the I/Q modulation (which feed the D/A conversion) are read and validated;

(3) these reference signals are used to test the Rx modules. They are stored in an internal memory of the platform and processed by the Rx substituting real received data. This way, it is ensured that no interference due to D/A or $\mathrm{A} / \mathrm{D}$ conversion is present. Processing results of the $\mathrm{Rx}$ baseband blocks are read and compared to the results of bit-accurate simulations.

The baseband loop-back allows to check the interoperation of the Tx and Rx without requiring any RF front-end. Nevertheless, a simple connection of the Tx outputs to the Rx inputs is insufficient since neither of the analog front-ends deal 
TABLE 1: Summary of fixed-point variables precision at the Rx.

\begin{tabular}{lc}
\hline Variable & Number of bits $\left[b_{I} \cdot b_{D}\right]$ \\
\hline AGC output & {$[2.9]$} \\
Digital I/Q output & {$[3.8]$} \\
Antialiasing filter output & {$[3.8]$} \\
CIR estimate coefficients & {$[2.6]$} \\
FFT input & {$[2.12]$} \\
FFT output & {$[2.6]$} \\
Demapper distance metric & 10 \\
\hline
\end{tabular}

with I/Q (de)modulation. Thus, digital I/Q (de)modulation at an IF of $20 \mathrm{MHz}$ is implemented on the baseband platform using an $80 \mathrm{MHz}$ sampling clock. Hence, the real-valued signal after the $\mathrm{D} / \mathrm{A}$ conversion is centered around $20 \mathrm{MHz}$ with a $20 \mathrm{MHz}$ bandwidth.

An extensive evaluation of the precision of the different variables involved at the receiver side was performed using a fixed-point $\mathrm{C}++$ simulator. First, each variable is isolated by assuming that the rest has full precision. The number of bits used for the fixed-point representation is then adjusted in order to avoid error propagation and have reasonable performance degradation. We summarize in Table 1 the resolution of the different variables used in the final platform. The values are expressed as $\left[b_{I} \cdot b_{D}\right]$, where $b_{I}$ (resp., $\left.b_{D}\right)$ is the number of bits for the integer (resp., decimal) part. The overall variable is represented with $1+b_{I}+b_{D}$ bits, including the sign bit.

\subsection{Overall testbed performance: measurements in the wireless setup}

The wireless testbed was operated in our lab, within a demonstration scenario aiming at emphasizing the performance improvement brought by multiple antennas in the WLAN context. The exact configuration of the demonstration scenario is depicted in Figure 15, with the AP on the left and the MT on the right. Given the presence of lab furniture, it was expected that the propagation channel contained multiple separable paths. Measurements of the MIMO propagation channel are plotted in Figure 16, both in frequency domain and time domain. The two curves are the first channel response estimated during a given data acquisition period, and the last channel response of the same acquisition period, leading to the computation of a given bit error rare (BER) value. Figure 16 highlights several important characteristics of the propagation at hand in our demonstration context:

(i) during the data acquisition phase leading to the computation of a given BER value, the channel is static;

(ii) the MIMO propagation channel encountered is frequency selective, with attenuations up to $15 \mathrm{~dB}$ from one carrier to another (power measurements);

(iii) the multipath does not create any additional interblock interference, since it is completely absorbed by the 16 -sample ( 800 nanoseconds) guard interval;

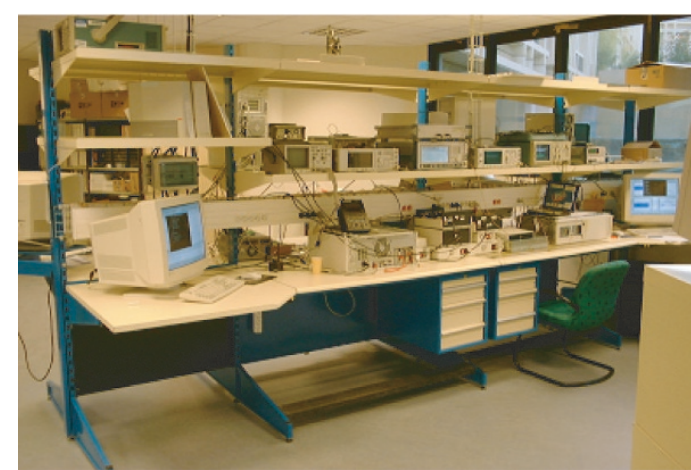

FIGURE 15: Demonstration scenario for BER measurements.

however, this guard period is not over-dimensioned for our scenario;

(iv) the 4 subchannels display very different characteristics.

The BER was measured with respect to the transmitted power for all supported antenna configurations, and the benefits of the proposed MIMO extensions appear clearly: up to $14.5 \mathrm{~dB}$ gain is measured for the $2 \mathrm{Tx}, 2 \mathrm{Rx}$ configuration with respect to the reference single-antenna setup. These results have been measured for a 16-QAM constellation and coding rate $1 / 2$, which corresponds to a data rate of $24 \mathrm{Mbps}$, and they are presented in Figure 17. Some comments on these results are in order. First of all, the performance improvement when going from the $2 \times 1$ (MISO) configuration to the $1 \times 2$ (SIMO) configuration is $3 \mathrm{~dB}$, as expected. Second, a large reduction in Tx power required can be expected at a given BER level, if a large difference in slope is observed. Now, the slopes at the bottom part of Figure 17 appear to be quite similar for the different cases. It may be that this impression arises due to the possibility that the implemented receiver only starts to work well at lower BER. This is an effect that would raise the curves at higher BER levels, creating a steeper slope for the lower BER levels. Also, as mentioned earlier, each point on these curves has been obtained with a different channel realization, that probably did not move much over the 20 frames of data gathered to produce each point. This lack of averaging leads to some variations in the curves. It should be emphasized that the results obtained depend on the positioning of the antennas of MT and AP, though the results reported in Figure 17 correspond to a random positioning, that has not at all been chosen so as to lead to maximum gain. Several other positions have been tried, with less extensive measurement results, and in one other position a gain of $9 \mathrm{~dB}$ was observed.

\section{CONCLUSIONS}

The goal of the OFDM WLAN testbed developed and discussed here was to demonstrate the improvement brought by adding the MIMO dimension to standard WLAN techniques. The design of the MIMO testbed was the result of a careful analysis of and interaction between propagation studies, algorithm development, and antenna design. The propagation 

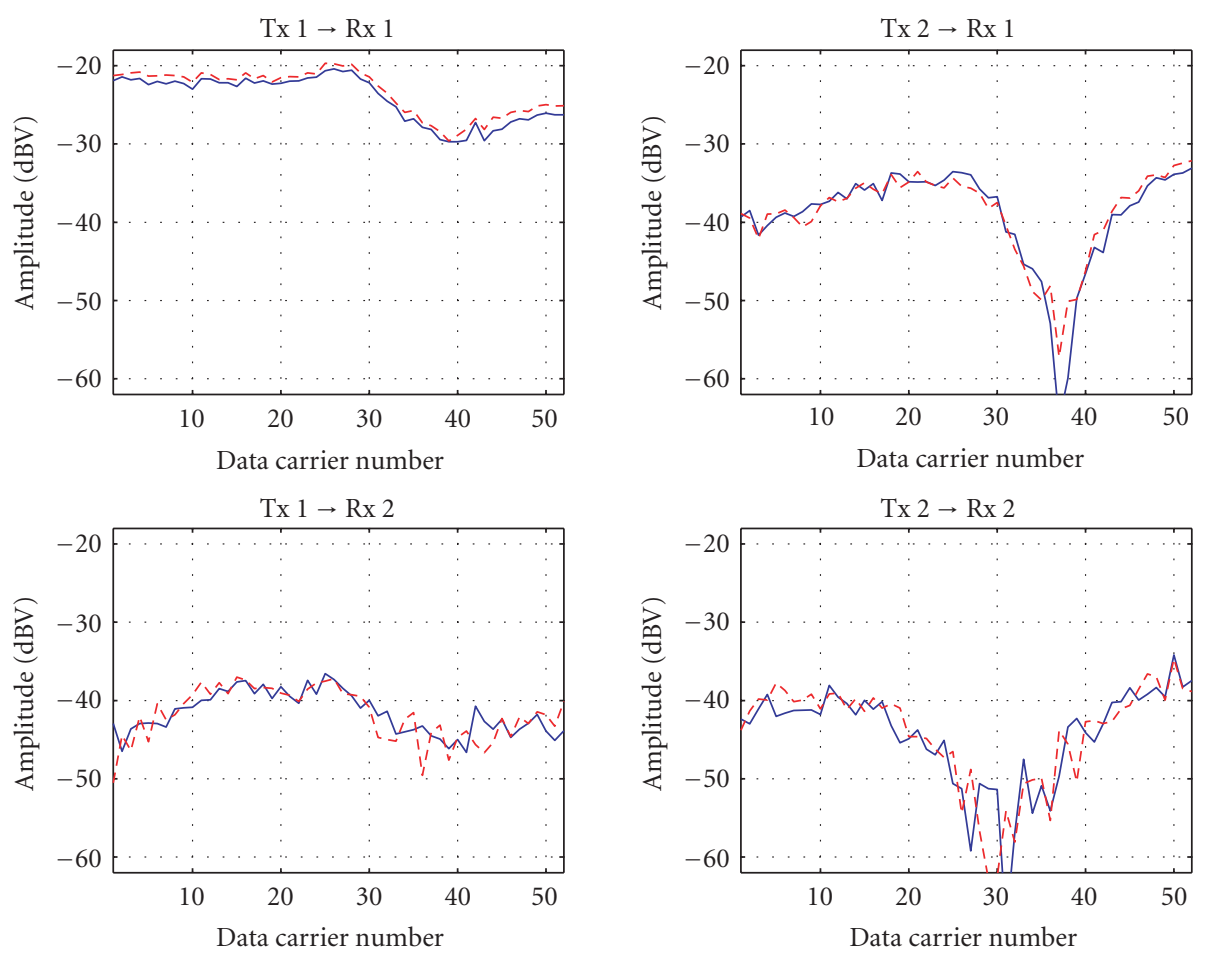

(a)
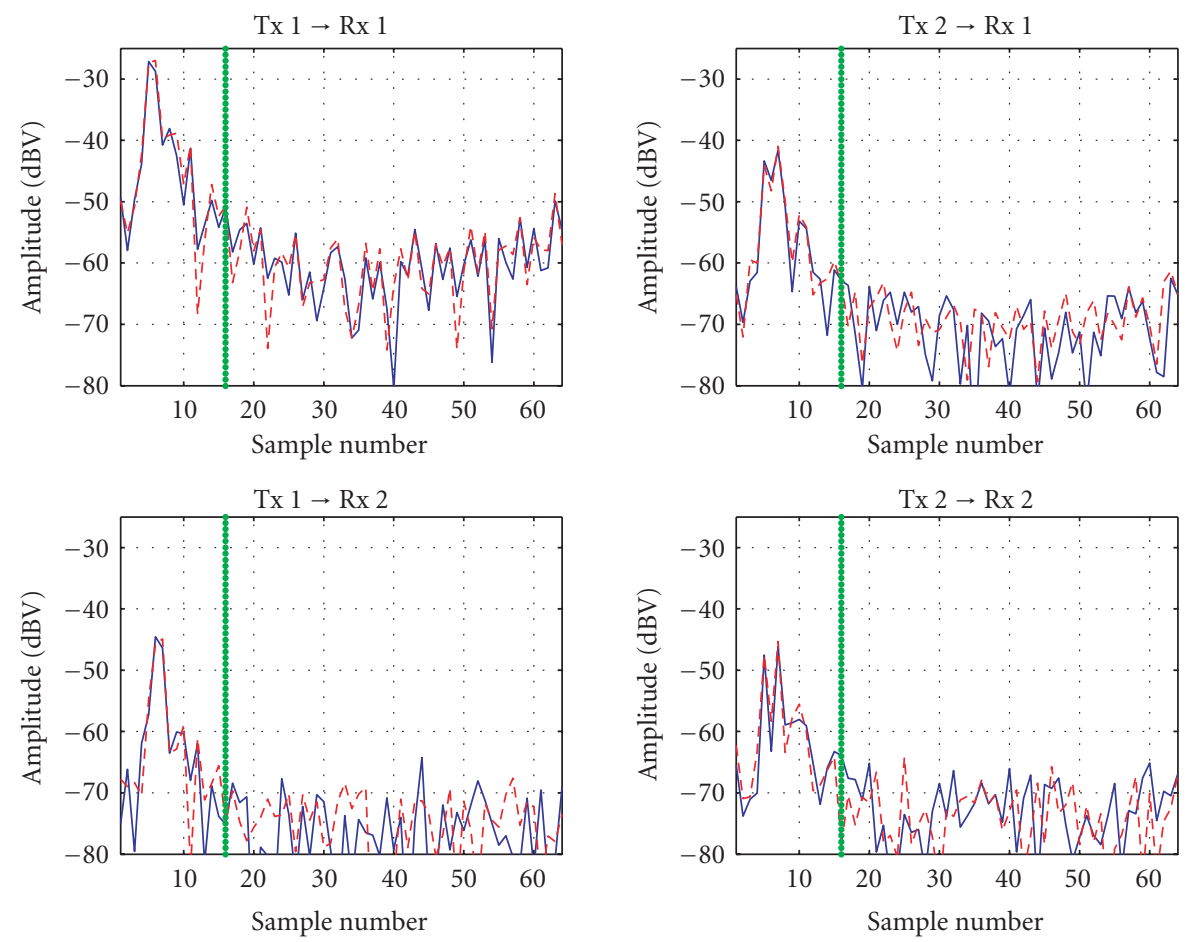

(b)

FIGURE 16: Frequency response and time impulse response of measured realizations of the MIMO propagation channel. (a) Frequencydomain observation. (b) Time-domain observation. 


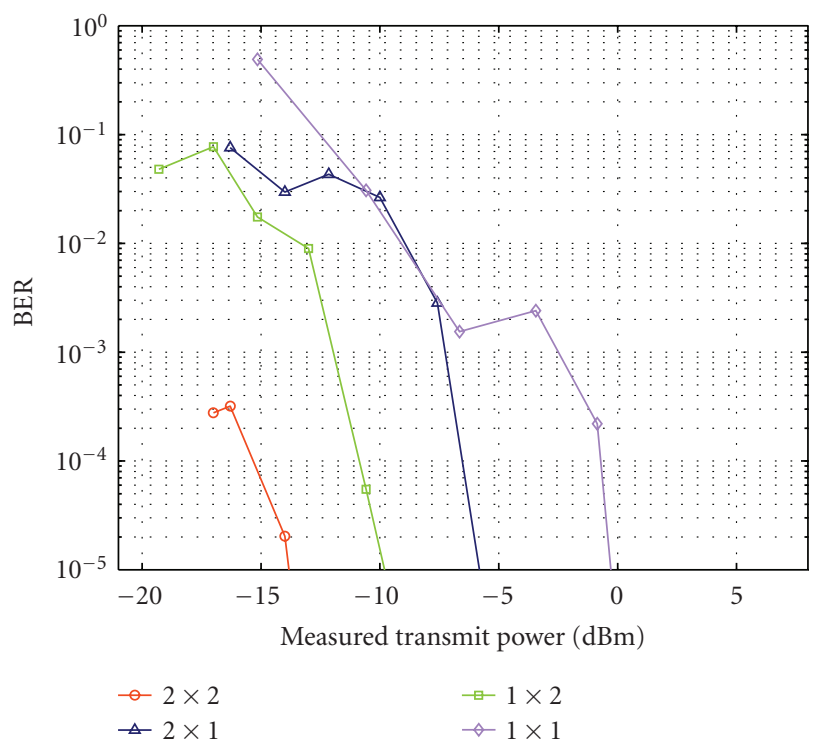

FIGURE 17: BER measured at $24 \mathrm{Mbps}$ for the four antenna configurations versus measured transmit power, 16 QAM symbols.

studies for an indoor environment showed that sufficient spatial diversity is present (at least at the MT side) to be able to design algorithms as for the (spatially) i.i.d. Rayleigh case, for limited MIMO configurations such as $2 \times 2$ or $4 \times 4$. The frequency selectivity on the other hand, even though nonnegligible, turned out to be quite limited, over the frequency band considered.

These considerations have been taken into account for the design of innovative space-time coding solutions. To this end, a wide range of space-time coding algorithms has been considered and compared. Algorithms can be classified in terms of the number of streams they transmit (degree of spatial multiplexing) and the degree of channel knowledge that gets exploited at the transmitter. Another aspect is the degree of diversity that can be obtained but this is normally maximal for the algorithms of interest. Innovative multistream algorithms have been considered for the case of no channel knowledge at the transmitter. In particular, ST-BICM has shown slightly better performance in the $2 \times 2$ case and significantly better performance for larger systems and rates. It has been observed that the addition of linear precoding leads to negligible improvement, once channel coding is present.

On the other hand, it was found that channel knowledge at the transmitter leads to little capacity gain at high SNR. But this high-SNR regime only materializes at higher SNR as the channel conditioning deteriorates. Single-stream solutions do not benefit from spatial multiplexing, but the ensuing loss was found to be very small for the $2 \times 2$ configuration of the testbed. So, for this $2 \times 2$ case, it was found that the Alamouti algorithm was close to optimal for the bit rates considered. When moving to larger MIMO systems, such as the $4 \times 4$ case, single-stream solutions become largely suboptimal. Also multistream solutions that do not benefit from maximal diversity, such as V-BLAST, become quite suboptimal. The ST-BICM approach discussed here then becomes very competitive.

The propagation studies also lead to appropriate antenna designs. At the AP, as the angular aperture is limited, a solution based on a classical antenna array has been proposed to exploit the spatial diversity. At the MT, where the spatial diversity is mainly due to angular diversity, pattern diversity was used. Furthermore, some research was performed into the optimization of pattern diversity for a larger number of antennas with practical antenna designs.

The measured results obtained with the $2 \times 2$ testbed confirmed the algorithmic choices made on the basis of analysis and simulations. In particular, the simple Alamouti code with standard channel coding and simple channel estimation provides impressive improvements for the $2 \times 2$ configuration over the $1 \times 1$ configuration in terms of robustness to the placement of receive and transmit antennas and general BER. This improved performance can be exploited to increase bit rate and/or decrease Tx power and/or increase range. Also, patch antennas were found to constitute a satisfactory lowcost solution, allowing for the integration of active RF components close to the antennas at the MT.

The developed testbed demonstrates that interesting MIMO performance can be obtained with readily available hardware components, reasonable wordlengths, and overall complexity. Experience with the testbed shows that the choice of antenna array (pattern and, e.g., polarization diversity) is a critical issue and that the algorithms may be usefully adapted to the considered environment.

\section{ACKNOWLEDGMENTS}

The research leading to this publication was partially supported by the French National Telecommunications Research 
Network (RNRT) project ANTIPODE, by the IST FITNESS project, and by Antennessa, France Télécom R\&D, Eurécom Institute, Motorola, and ENST Bretagne.

\section{REFERENCES}

[1] I. Emre Telatar, "Capacity of multi-antenna Gaussian channels," European Transactions on Telecommunications, vol. 10, no. 6, pp. 585-595, 1999.

[2] G. J. Foschini and M. J. Gans, "On limits of wireless communications in a fading environment when using multiple antennas," Wireless Personal Communications, vol. 6, no. 3, pp. 311-335, 1998.

[3] P. Laspougeas, J.-M. Conrat, S. Mayrargue, and P. Pajusco, "Single and dual multi-sensor channel characterisation in Indoor and Campus environments at $5 \mathrm{GHz}$," in Proceedings of IST Mobile Communications Summit, Barcelona, Spain, September 2001.

[4] ETSI Normalization Committee, "Channel models for HIPERLAN/2 in different indoor scenarios," Norm ETSI doc. 3ERIO85B, European Telecommunications Standards Institute, Sophia-Antipolis, France, 1998.

[5] P. Laspougeas, P. Pajusco, and J.-C. Bic, "Spatial radio channel model for UMTS in urban small cells area," in Proceedings of European Conference on Wireless Technology (ECWT '00), pp. 118-121, Paris, France, October 2000.

[6] P. Laspougeas, P. Pajusco, and J.-C. Bic, "Radio propagation in urban small cells environment at $2 \mathrm{GHz}$ : experimental spatio-temporal characterization and spatial wideband channel model," in Proceedings of 52nd IEEE Vehicular Technology Conference (VTC '00), vol. 2, pp. 885-892, Boston, Mass, USA, September 2000.

[7] J.-M. Conrat and P. Pajusco, "A versatile propagation channel simulator for MIMO link level simulation," COST 273 Project Report TD(03)120, Paris, France, 2003.

[8] V. Erceg, L. Schumacher, P. Kyritsi, et al., "IEEE P802.11 Wireless LANs: TGn Channel Models," IEEE 802.11 document 03/940r1, 2003.

[9] L. Chaigneaud, V. Guillet, and R. Vauzelle, "A 3D ray-tracing tool for broadband wireless systems," in Proceedings of IEEE VTS 54th Vehicular Technology Conference (VTC '01), vol. 4, pp. 2043-2047, Atlantic City, NJ, USA, October 2001.

[10] H. Bölcskei, M. Borgmann, and A. J. Paulraj, "Impact of the propagation environment on the performance of spacefrequency coded MIMO-OFDM," IEEE Journal on Selected Areas in Communications, vol. 21, no. 3, pp. 427-439, 2003, Special issue on MIMO systems and applications.

[11] D. Gesbert, H. Bölcskei, D. Gore, and A. J. Paulraj, "MIMO wireless channels: capacity and performance prediction," in Proceedings of IEEE Global Telecommunications Conference (GLOBECOM '00), vol. 2, pp. 1083-1088, San Francisco, Calif, USA, November-December 2000.

[12] D. Chizhik, G. J. Foschini, M. J. Gans, and R. A. Valenzuela, "Keyholes, correlations, and capacities of multielement transmit and receive antennas," IEEE Transactions on Wireless Communications, vol. 1, no. 2, pp. 361-368, 2002.

[13] S. Visuri and D. T. M. Slock, "Colocated antenna arrays: design desiderata for wireless communications," in Proceedings of 2nd IEEE Sensor Array and Multichannel Signal Processing
Workshop (SAM '02), pp. 580-584, Rosslyn, Va, USA, August 2002.

[14] S. M. Alamouti, "A simple transmit diversity technique for wireless communications," IEEE Journal on Selected Areas in Communications, vol. 16, no. 8, pp. 1451-1458, 1998.

[15] O. Tirkkonen, A. Boariu, and A. Hottinen, "Minimal nonorthogonality rate 1 space-time block code for $3+$ Tx antennas," in Proceedings of IEEE 6th International Symposium on Spread Spectrum Techniques and Applications, vol. 2, pp. 429432, Parsippany, NJ, USA, September 2000.

[16] P. W. Wolniansky, G. J. Foschini, G. D. Golden, and R. A. Valenzuela, "V-BLAST: an architecture for realizing very high data rates over the rich-scattering wireless channel," in Proceedings of URSI International Symposium on Signals, Systems, and Electronics (ISSSE '98), pp. 295-300, Pisa, Italy, September-October 1998.

[17] M. Guillaud and D. T. M. Slock, "Full-rate full-diversity spacefrequency coding for MIMO OFDM systems," in Proceedings of 3rd IEEE Benelux Signal Processing Symposium (SPS '02), Leuven, Belgium, March 2002.

[18] M. Guillaud and D. T. M. Slock, "Multi-stream coding for MIMO OFDM systems with space-time-frequency spreading," in Proceedings of 5th International Symposium on Wireless Personal Multimedia Communications (WPMC '02), vol. 1, pp. 120-124, Honolulu, Hawaii, USA, October 2002.

[19] J. Boutros, N. Gresset, and L. Brunel, "Turbo coding \& decoding for multiple-antenna channels," in Proceedings of $3 \mathrm{rd} \mathrm{In-}$ ternational Symposium on Turbo Codes and Related Topics, pp. 185-186, Brest, France, September 2003.

[20] A. Guillén i Fàbregas and G. Caire, "Impact of signal constellation expansion on the achievable diversity in quasistatic multiple-antenna channels," in Proceedings of 12th European Signal Processing Conference (EUSIPCO '04), Vienna, Austria, September 2004.

[21] A. Guillén i Fàbregas and G. Caire, "Design of space-time BICM codes for block fading channels with iterative decoding," in Proceedings of 37th Conference on Information Sciences and Systems (CISS '03), Johns Hopkins University Press, Baltimore, Md, USA, March 2003.

[22] E. Biglieri, G. Caire, and G. Taricco, "Limiting performance of block-fading channels with multiple antennas," IEEE Transactions on Information Theory, vol. 47, no. 4, pp. 1273-1289, 2001.

[23] R. Knopp and G. Caire, "Power control and beamforming for systems with multiple transmit and receive antennas," IEEE Transactions on Wireless Communications, vol. 1, no. 4, pp. 638-648, 2002.

[24] V. Tarokh, H. Jafarkhani, and A. R. Calderbank, "Space-time block codes from orthogonal designs," IEEE Transactions on Information Theory, vol. 45, no. 5, pp. 1456-1467, 1999.

[25] A. R. Dias, S. Rouquette, and K. Gosse, "MTMR channel estimation and pilot design in the context of space-time block coded OFDM-based WLANs," in Proceedings of IST Mobile and Wireless Telecommunications Summit, pp. 73-77, Thessaloniki, Greece, June 2002.

[26] D. T. M. Slock, "Signal processing challenges for wireless communications," in Proceedings of 1st International Symposium on Control, Communications and Signal Processing (ISCCSP '02), pp. 881-892, Hammamet, Tunisia, March 2004. 
Albert Guillén i Fàbregas was born in Barcelona, Spain, in 1974. He received the Telecommunications and Electronics Engineering degrees from Universitat Politecnica de Catalunya, Barcelona, Spain, and the Politecnico di Torino, Torino, Italy, respectively, in 1999, and the Ph.D. degree in communication systems from École Polytechnique Fédérale de Lausanne (EPFL), Lausanne, Switzerland, in 2004. He conducted

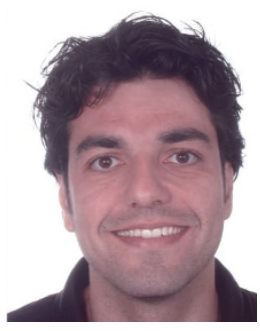
his final research project at the New Jersey Institute of Technology (NJIT), Newark, NJ. He has been with the Telecom Italia Research Laboratories, Torino, Italy, and with the European Space Agency (ESA), Noordwijk, The Netherlands. From 2001 to 2004, he was a research and teaching assistant at Institut Eurécom, SophiaAntipolis, France. From June 2003 to July 2004, he was a visiting scholar at EPFL. Since September 2004, he has been a Research Fellow at the University of South Australia, Mawson Lakes, Australia. His research areas are communication theory, information theory, coding theory, and signal processing. He received a Research Fellowship of the Spanish Ministry of Education to join ESA. He received the Young Authors Award of the 2004 European Signal Processing Conference EUSIPCO 2004, Vienna, Austria, and the 2004 Nokia Best Doctoral Thesis Award from the Spanish Institution of Telecommunications Engineers.

Maxime Guillaud was born in BourgoinJallieu, France, in 1977. He received the Engineer degree in electronics from École Nationale Supérieure de l'Electronique et de ses Applications (ENSEA), Cergy, France, in 2000, and the Ph.D. degree in electrical engineering from École Nationale Supérieure des Télécommunications (ENST), Paris, France, in 2005. From 2000 to 2001, he was a Research Engineer at Lucent Technologies'

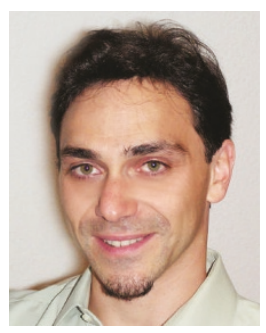
Bell Laboratories, Holmdel, NJ, USA, where he worked on the prototyping of MIMO UMTS systems. In 2001, he joined the Mobile Communications Department of Eurécom Institute, SophiaAntipolis, France. There, he focused on space-time coding and channel modeling for channels with many degrees of freedom. $\mathrm{He}$ also investigated channel reciprocity and the associated calibration issues, and introduced the concept of relative calibration. He is the recipient of a SPAWC '05 Student Paper Award.

Dirk T. M. Slock received an Engineering degree from the University of Gent, Belgium, in 1982. In 1984 he was awarded a Fulbright scholarship from Stanford University, where he received the M.S. degree in EE, M.S. degree in statistics, and Ph.D. degree in EE in 1986, 1989, and 1989, respectively. While at Stanford, he developed new fast RLS algorithms for adaptive filtering. During 1989-91, he was with Philips Research Belgium. In 1991, he joined the Eurecom Institute where he is now Professor. He teaches and does research on statistical signal processing and its application to wireless communications. He invented semiblind equalization, single-antenna interference cancellation for GSM, and the equalizer-correlator receiver now used in 3G WCDMA terminals.
In 2000, he cofounded SigTone, a start-up developing music DSP product. He has also been a consultant on xDSL and DVB-T systems. He received one Best Journal Paper Award from the IEEESP and one from EURASIP in 1992. He is the coauthor of two IEEE Globecom98, one IEEE SIU'04, and one IEEE SPAWC'05 Best Student Paper Award. He was an Associate editor for the IEEE-SP Transactions in 1994-96. He is an Editor for EURASIP JASP, for which he also guest edited two special issues. He is a Fellow of the IEEE.

Giuseppe Caire was born in Torino, Italy, in 1965. He received the B.S. degree in electrical engineering from Politecnico di Torino (Italy), in 1990, the M.S. degree in electrical engineering from Princeton University in 1992, and the Ph.D. degree from Politecnico di Torino in 1994. He was a recipient of the AEI G. Someda Scholarship in 1991. He has been with the European Space Agency (ESTEC, Noordwijk, The Netherlands) from

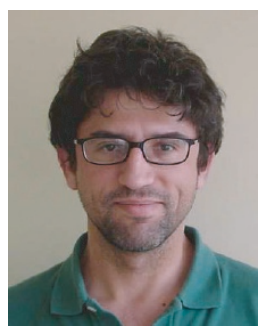
May 1994 to February 1995. Also, he has been an assistant Professor in telecommunications at the Politecnico di Torino, Associate Professor at the University of Parma, Italy, Professor at the Institute Eurecom, Sophia-Antipolis, France, and he is presently Professor with the Viterbi School of Engineering, University of Southern California, Los Angeles, Calif He received the Jack Neubauer Best System Paper Award from the IEEE Vehicular Technology Society in 2003, the Joint IT/Comsoc Best Paper Award in 2004, and he is a Fellow of IEEE.

Karine Gosse graduated from the École Nationale Supérieure des Télécommunications in 1993, and she got her Ph.D. degree in digital signal processing from the ENST in 1996. Since then, she has been working with Motorola Labs in France, on digital communication systems design, focussing between 1999 and 2004, as Team Leader, on multiantenna signal processing approaches for short-range communication

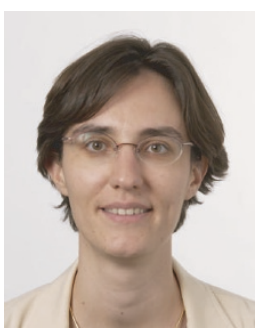
systems. Her areas of interest include digital signal processing and digital communications, multicarrier modulation, spatial diversity, and multiplexing techniques.

Stéphanie Rouquette was born in Figeac, France, in 1973. She received the Engineering degree from the École Nationale Supérieure d'Electronique et de Radioélectricité de Bordeaux (ENSERB), Talence, France, in 1995. She received the Ph.D. degree from ENSERB in 1998, where she worked on 2D high-resolution frequency estimation and radar imaging. In 1999, during a postdoctoral year at the Commissariat

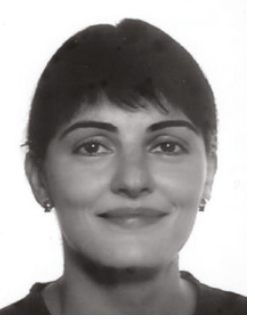
à l'Energie Atomique (CEA), Saclay, France, she applied signal processing techniques to the functional analysis of magnetic resonance images. She then joined Motorola Labs, Paris, France, where she is currently a Senior Research Engineer. Her research interests include adaptive antennas for wireless communications, OFDM modulation, and channel coding. 
Alexandre Ribeiro Dias was born in Paris, France, in 1975. He received an Engineering degree in digital signal processing and system engineering from École Nationale Supérieure des Télécommunications, Paris, France, in 2001. Recruited by Motorola Laboratories, Paris, in 2001, his research interests were digital signal processing for MIMO systems. He was involved in the WLAN standardization activ-

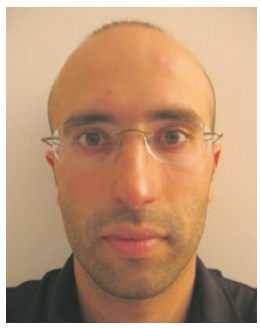
ities of IEEE802.11n, where he made several contributions for MIMO technologies. He joined Motorola's Mobile Devices Business, Toulouse, France, in 2004, where he is currently a Senior Software Engineer working on validation and conformance testing of GSM/EGPRS cellular phones for new features such as DARP.

Philippe Bernardin graduated from the École Supérieure d'Ingénieurs en Electronique et Electrotechnique, Marne la Vallée, France, in 1998, in the specialty of digital communication systems. After receiving his degree, he first worked in the Radar Research Department, THALES. He joined the Motorola Research Center in 2000. Since then, he has been contributing to wireless physical layer research studies and particu-

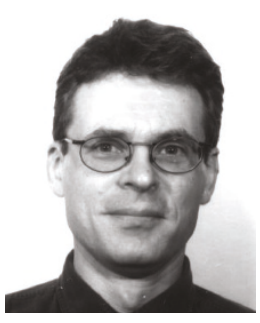
larly worked on real-time hardware implementation. Before 1994, he was Project Leader in the area of professional phone equipment and particularly he studied and built real-time multispeakers speech recognition systems between 1989 and 1994, in collaboration with the Centre National dÉtude des Télécommunications, France Telecom. In particular he developed the first commercial system of speech recognition installed in France for the insurance company La MACIF. This first activity followed his degree received from the University of Creteil, France, in 1985.

Xavier Miet received the Engineering degree in electrical engineering and digital signal processing from École Nationale Supérieure d'Electronique, Informatique et Radiocommunications, Bordeaux, France, in 1999 and the Master of Science degree in digital design and digital communication from École Nationale Supérieure des Télécommunications, Paris, France, in 2000. Recruited by Motorola Laboratories

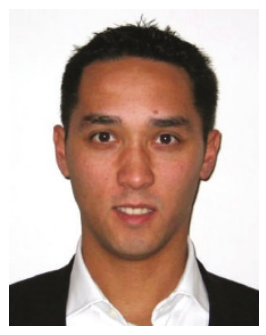
in November 2000, he is currently a Senior Research Engineer. His recent research interests include VLSI for digital wireless communication with particular focus on emerging WLAN technologies.

Jean-Marc Conrat was born in Nancy, France, in 1968. He received the M.S. degree in electrical engineering from the Applied Science National Institut (INSA), Lyon, France, in 1991. Since 1993, he has been with France Télécom Research and Development, Belfort, France. He is involved in propagation channel measurements, modeling, and simulation. His current research interests are in the modeling of the direc-

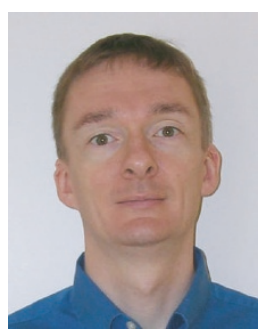
tional wideband propagation channel for MIMO applications.
Yann Toutain received the Engineer degree from the École Nationale Supérieure de Télécommunication de Bretagne, Brest, France, in 1997. Then he received his Ph.D. degree in electronics from ENSTBr and Occidental Brittany University, Brest, France, in 2001. He joined Antennessa, Brest, France, in 2001 where he spent three years working on antenna development for the telecom, automotive, military, and other specialized markets. He is currently the head of the Dosimetry Department, Antennessa.

Alain Peden received the Diplôme d'Etudes Supérieures Spécialisées degree from the University of Limoges, France, in 1985 . He was an MMIC Designer at Thomson Composants Microondes from 1986 to 1988 . He joined the Laboratory of Electronics and Systems for Telecommunications (LEST), École Nationale Supérieure des Télécommunications in Brest, France, in 1989 and received the Ph.D. degree in electronics in

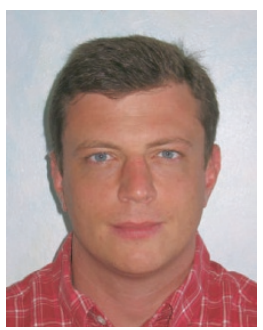
1991. He is currently an Associate Professor and his research activities are in the field of microwave and millimetre wave power amplifier design. He is mainly involved with the large-signal characterization of transistors and nonlinear devices and applications to amplifier design using power combining and quasioptical techniques.

Zaiqing Li was born in Shandong, China, in 1967. He received the Bachelor's degree and Master's degree in electrical and communication engineering from Harbin Institute of Technology, in 1989 and 1992, respectively. In 1992, he joined the Department of Electrical and Communication Engineering, Harbin Institute of Technology, China. From 2000 to 2001, he was a visiting scholar in the Laboratory of Electron-

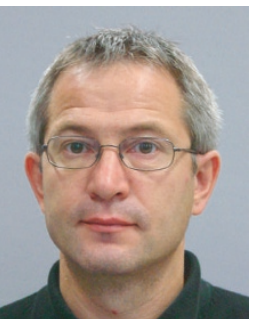
ics and Systems for Telecommunications (LEST), Brest, France. He is currently finishing his Ph.D. degree at the National Engineer Graduate School (ENST-Bretagne), France. His research interests involve computational electromagnetics and microwave/millimeter circuits.

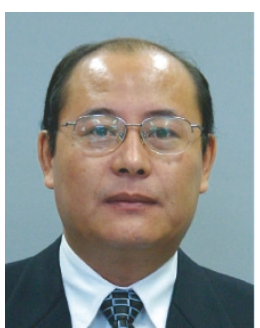

